\title{
Colors of Autism Spectrum: A Single Paradigm Explains the Heterogeneity in Autism Spectrum Disorder
}

\author{
András Lőrincz \\ Faculty of Informatics \\ Eötvös Loránd University \\ Budapest \\ Hungary
}

\begin{abstract}
We propose a theory of ASD as a condition of comorbid cognitive impairments that corrupt the learning, encoding, and manipulation of episodic and semantic memories. We consider (i) episodic and semantic memory functions of the entorhinal-hippocampal complex, (ii) constraints on the transfer and encoding of these memory components into neocortical areas, and (iii) the demands of cognitively manipulating memories in distributed computations being necessary for goaloriented interactions. In ASD, learning and cognitive challenges manifest in diverse ways but especially in high-complexity-model predictive control tasks with latent variables. ASD impairments in social interactions represent a prototypical example. Social interactions are at the high end of complexity and require processes (i) - (iii) to work in a concerted fashion due to the need for the learning and estimation of many, sometimes latent, parameters, including emotions, intention, physical and mental capabilities as well as the predictive modeling of these parameters for decision making and timed-action series. We put forth the idea that autism is a result of an arbitrary combination of otherwise not prominent corruptions in processes (i)(iii). Together, these corruptions may severely impair intelligence and slow down learning, especially in highcomplexity learning tasks. Over time, slow learning may spare the spontaneous learning-by-doing method - namely, repetitive behavioral patterns, whereas behavioral failures related to complex tasks can restrict interest in such task, thus inducing a fear of novelty; conversely, the fear of novelty restricts interest and
\end{abstract}

can slow learning down. We embed our thoughts into a predictive autoencoding, goal-oriented model of a deterministic world. We compare this model to others, such as the noisy brain model, the Bayesian prior theory, the mirror neuron theory and the weak central coherence theory. We argue that the predictive autoencoder model of the deterministic world harmonizes with these other models and embraces them in a straightforward way.

\section{AUTHOR SUMMARY}

The core of our arguments is based on the autoencoder model of the entorhinal-hippocampal complex and its role in forming declarative memories. We argue that social behavior and language usage are highly complex cognitive tasks involving the encoding, learning, and manipulation of declarative memories with latent variables. All these processes can be corrupted in many ways. Corruptions may manifest as epilepsy, schizophrenia, attention deficit, and depression, among other disorders. However, if these symptoms are weak but co-occur, then the most strongly affected behavioral feature is the ability to solve complex cognitive tasks. This condition can be brought about by diverse combinations of impairments that sum up in corrupted social behavior the most.

\section{KEYWORDS}

autism, cognition, components, psychiatric impairments, comorbidity 
Colors of Autism Spectrum:

\section{Contents}

1 Introduction 3

2 Theoretical background in the context of experimental findings 4

2.1 Components: an overview of the concept $\ldots \ldots \ldots \ldots \ldots \ldots$

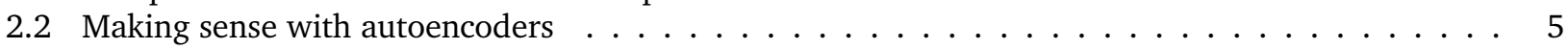

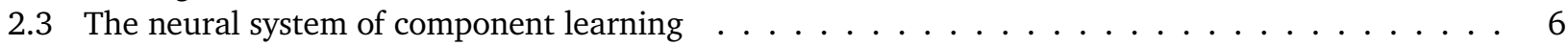

2.3.1 The discretization of the allothetic space component $\ldots \ldots \ldots \ldots \ldots$

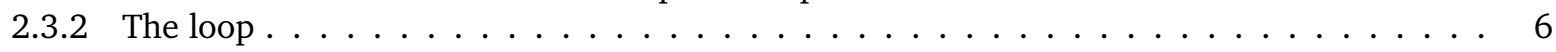

2.3 .3 Episodic memory from temporal relations $\ldots \ldots \ldots \ldots \ldots \ldots \ldots \ldots \ldots \ldots \ldots$

2.3 .4 Forming semantic memory by factoring out time $\ldots \ldots \ldots \ldots \ldots \ldots$

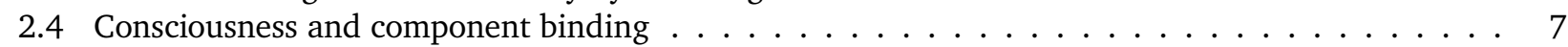

2.5 Two-stage operation: Memory encoding and consolidation . . . . . . . . . . . . . . . . 8

2.5 .1 Brain waves contribute to memory consolidation in many ways $\ldots \ldots \ldots \ldots$

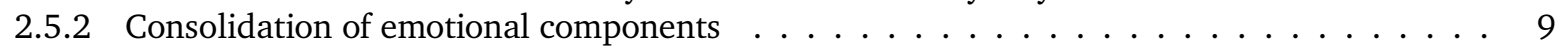

2.6 Bottom-up and top-down processing serves component formation $\ldots \ldots \ldots \ldots$

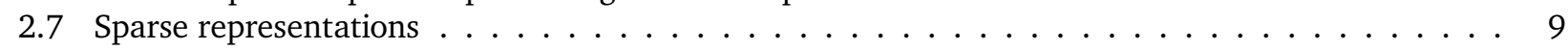

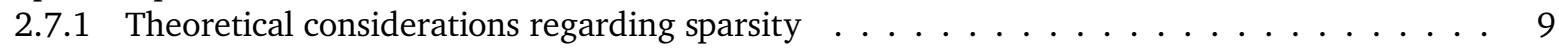

2.7 .2 Constraints and errors in sparse representations . . . . . . . . . . . . . . 10

2.7 .3 Autoencoding and interpretability . . . . . . . . . . . . . . . . . 10

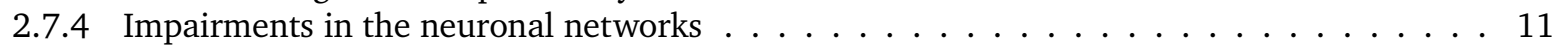

2.7 .5 Brain structure impairments . . . . . . . . . . . . . . . . . . . 11

2.7.6 Differences in axial diffusivity, myelination, and synaptic homeostasis . . . . . . . . . . . 11

2.8 Summary of some phenomena that may impair component learning and cognitive manipulations . 12

3 'Results': Falsifying issues 13

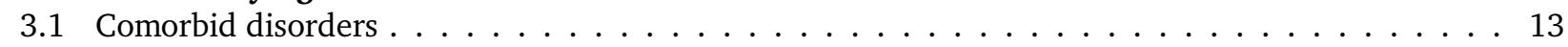

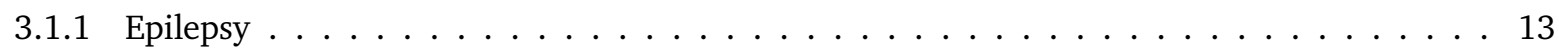

3.1 .2 Schizophrenia . . . . . . . . . . . . . . . . . . . . . . 15

3.1 .3 Attention deficit, hyperactivity disorder $\ldots \ldots \ldots \ldots \ldots \ldots \ldots$

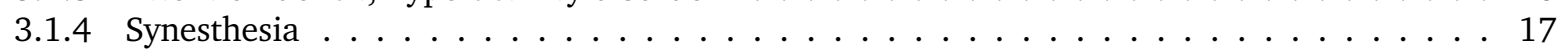

3.2 Environmental effects: Discordant monozygotic twins . . . . . . . . . . . . . . . . . . . 17

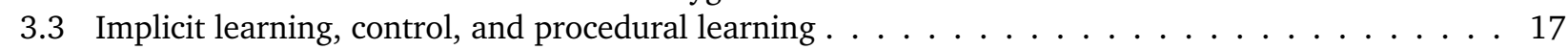

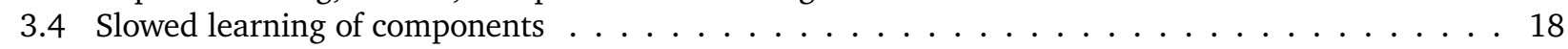

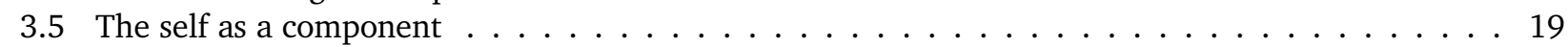

4 Discussion: Comparisons with other models 19

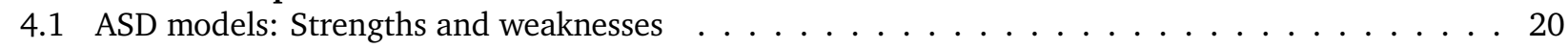

4.1 .1 Noisy brain and excitation/inhibition ratio imbalance . . . . . . . . . . . . 20

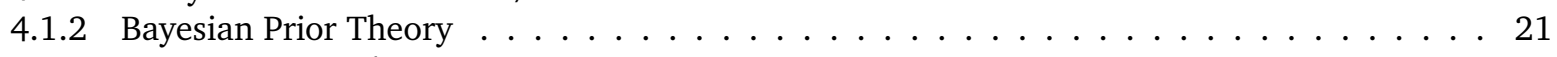

4.1 .3 Mirror Neuron Theory . . . . . . . . . . . . . . . . . . . . . . . . . . 21

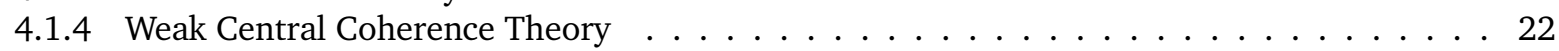

4.1 .5 Going deeper: Specific models . . . . . . . . . . . . . . . . . . . . 22

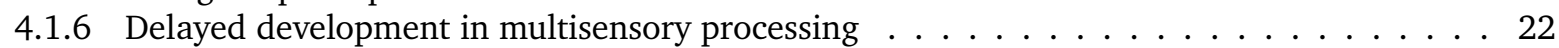

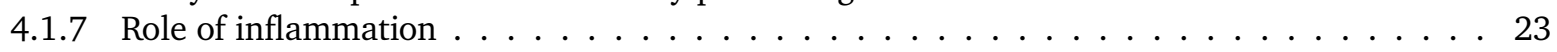

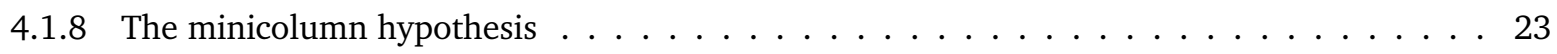

4.1.9 Cortical hyperexpansion, brain volume overgrowth, and spatio-temporal constraints . . . 23

4.1 .10 Malfunctioning interneurons . . . . . . . . . . . . . . . . . . 24

4.1 .11 Cognitive dysfunction $\ldots \ldots \ldots \ldots \ldots \ldots \ldots \ldots$

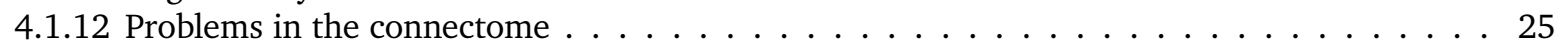

4.2 Potential side effects of impairments of component formation $\ldots \ldots \ldots \ldots$

5 Conclusions 
Colors of Autism Spectrum:

\section{Introduction}

Below, we consider some arguments to formulate our single paradigm hypothesis on the origin of autism. This single paradigm concerns the complexity of computations being dependent on the number of variables that gives rise to a search space scaling with that number in the exponent. The base of the exponent is known to be less relevant, and we set it to 2 for the sake of simplicity.

There is an astonishing fact, a peculiar feature of human knowledge: It takes approximately 20 years for an individual to learn a considerable portion of the available scientific knowledge that humankind has collected in the past 20,000 years. Thus, much less time is needed to communicate knowledge than to discover that knowledge. Since this is not a necessity in complexity theory, we ask how this might happen.

Consider the space of sensory information, the space of memory span, and the space related to languages. The sensory space is gigantic. For example, the number of variables perceived on the retina equals the number of neurons, which is on the order of one million. This defines the search space to be compared with the human memory span, which is between 5 and 9 items at one time. Thus, $2^{1,000,000}$ is the search space, and $\approx 2^{2}-2^{3}$ is the subspace that can be addressed at one time. This exponentiation of the variables is called the curse of dimensionality, a term coined by Richard Bellman approximately 60 years ago. It is hard to appreciate such numbers. To give an example, folding a piece of paper (only) 50 times would give rise to an object with thickness about as large as the Earth distance from the Sun.

One can communicate well with 2,000 , i.e., $\approx 2^{11}$ words. The space that language can cover is very large since sentences or documents can contain many words, and the number of words in a communication multiplies the exponent. A relatively short document may have 5,000 words; therefore, in principle, it is a member of space where the number of members is $2^{11 * 5,000}=2^{55,000}$. This temporal compositionality increases the expressive power of any language, including sign language and body 'language', for example. The meaning of any expression is ambiguous, and its context, i.e., the situation, the other expressions of the communication, the experiences of the partners individually or together, and the goal of the communication may disambiguate its meaning. Such disambiguation is also a cognitive task.

Another crucial matter is that our world, including the inertial motion of our body, to a good approximation, is deterministic. In turn, a deterministic model (a.k.a. a representation or interpretation) of the world should apply, including the inverse dynamics that control the body. However, the deterministic model differs from a Cartesian theater (Dennett, 1991) since it is a compressed approximation of the world, which is not fully observed. Ambiguities are present, and the brain must engage in a disambiguating process to select the best interpretation according to the available spatio-temporal context and act accordingly. Thus, the model should be predictive. However, delays in the processing are on the order of hundred milliseconds and must be overcome to match decision making with the control actions that follow. In addition, the related sensory signals may travel an additional hundred milliseconds or so before they can influence the model. In turn, decisions occur long before the deterministic model 'knows' about them. Sensory information about the outcomes has considerable delays, too. Nonetheless, consciously available information, i.e., the internal model, compensates for both in a way that makes it seem as if internal decision making and the related effects occur simultaneously, with a precision of a few milliseconds. Long-distance gamma synchronization and theta gamma wave interplay seem responsible for managing this outcome (Melloni et al., 2007).

Language is instructive for us since it offers a way to overcome the memory span bottleneck. Constraints posed by language give rise to certain conditions concerning learning, the execution of control and related constraints on communication:

1. Complex control programs spanning a longer time interval should be learned.

2. Means for launching learned control programs should exist.

3. The number of entities to be controlled independently at one time cannot be large.

4. Switches between control programs cannot be frequent.

These conditions may be satisfied if processes in the world can be segmented (separated) in space and time, i.e., if there are separable episodes.

Consider memory formation and memory manipulations. We have procedural (implicit) and declarative (explicit) memories. The latter are made of semantic and episodic memory forms; see, e.g., (Buzsáki and Moser, 2013)and the references therein. Declarative memory includes facts, rules, and autobiographic episodes, among other forms. These forms are first learned in the relatively small hippocampal-entorhinal complex (EHC). Then, they are transferred to neocortical areas for long-term storage. Learning, transfer and usage should satisfy the constraints posed by the needs of the predictive deterministic model. Uncertainties must last only a few milliseconds at each brain region to ensure timely actions despite the large delays in the distributed computational system of the brain.

The EHC is critical for learning, encoding, and transferring memories. The frontal lobe plays a central role in cognitive manipulations. These and the other brain regions compute together. Brain oscillations orchestrate and synchronize the computations involved in processing sensory information, launching actions and computing the deterministic representation. Nature has developed these brain oscillations. There are six 
Colors of Autism Spectrum:

different frequencies, which span the $0.1 \mathrm{~Hz}-100 \mathrm{~Hz}$ range, and their ratios have approximately the same values for all mammalian species, despite the large differences in brain size between mice and elephants, for example (Buzsáki and Watson, 2012).

The strict requirements for the computations in the brain can be corrupted in diverse ways. For example, the frequencies or strengths of brain oscillations may change, the amplitudes of these oscillations may go out of control, the wiring may be corrupted, and the selective procedure may be weak, to name a few possible types of corruption. Such corruptions have distinctive names, such as schizophrenia, epilepsy, microor macrocephaly, noisy brain, and others. However, if they are weak, but occur in combination, then the associated distinctive phenomena can corrupt learning, manipulation and the control of more complex tasks, such as language and social behavior. Social behavior, for example, involves dynamic internal and external inputs - at least two players, the target of the interaction, interests, goals, strategic thinking about reaching the goal, an understanding of the other player's feelings and beliefs, problem detection, and rapid decision making to seek solutions or compromises when partners face barriers. Additionally, internal factors, such as mood and motivation, may be influenced by other variables. The co-occurring impairments can corrupt any element on that list, and comorbidities can manifest in diverse ways. In our view, this is autistic spectrum disorder (ASD).

We shall proceed as follows. In the next section, we consider the problem of component forming. This is followed by our 'results', in which we specify disorders that can contribute to autism. In the discussion, we consider ASD models, what these models are missing, and why and how our model covers and complements them. We draw our conclusions and provide an outlook in the last section.

\section{Theoretical background in the context of experimental find- ings}

Let us start with basic thoughts on cognition and clarify our abstractions. We use the word 'cognition' to describe processes that manipulate and control a set of mental models equipped with features that represent past, ongoing, or planned interactions between them. Thus, features are related to the interaction, and features unrelated to the interaction or its goal can be neglected. A low-level example is eating; the choice of the object to be eaten depends on whether it has the feature 'edible'. Edibility is only one feature out of many, but it may be sufficient for decision-making and action at a given time. The feature 'edible' belongs to many objects, and many cognitive tasks may need to consider this feature. In other words, a feature may become a component in a cognitive task. The concept of component serves to define semantic memories, episodic memories, and the multi-scale relationships between them. We elaborate on the concept of components below.

\subsection{Components: an overview of the concept}

It is central to describe the concept of components, sometimes called factors, descriptors, or features, to explain why they are relevant and determine the criteria of a good descriptor. Another concern is how components can be applied in different goal-oriented contexts. As an illustration, consider the concept of numbers.

The central feature of number theory is generalization. When we use numbers, we strip off a large variety of descriptors and focus on the additivity property independent from the situation. Fig. 1 illustrates that the same equation may occur when planning a vacation or when considering a chemical reaction.

(a)
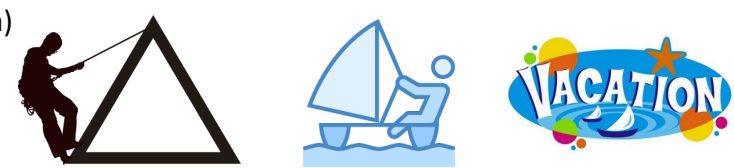

2 weeks (climbing) +1 week (sailing) $=3$ weeks (vacation) $2+1=3$

(b)

$$
\begin{gathered}
\mathrm{CaCl}_{2}+\mathrm{CO}_{2}+\mathrm{H}_{2} \mathrm{O} \Rightarrow \mathrm{CaCO}_{3}+2 \mathrm{HCl} \\
2 \text { oxygen atoms }+1 \text { oxygen atom }=3 \text { oxygen atoms } \\
2+1=3
\end{gathered}
$$

Figure 1: Numbers are special concepts.

Numbers are components and can be combined with other descriptors (components) in diverse ways. (a): Both climbing and sailing may be part of a vacation. (b): Oxygen is a constituent of carbon dioxide, water, and calcium carbonate

Numbers are special abstractions since they can be used diverse contexts, e.g., societal, biological, chemical and physical ones, but the other specific concepts of those disciplines are not needed for the derivations. Nonetheless, a number always corresponds to a feature and may lead to different conclusions in different disciplines. For example, the concept of distance is an additive quantity, and non-additivity leads to a special relativity. Additivity also applies to the Mendelejeev table, as in the chemical example in Fig. 1. Chlorine, for example, can play a role in many compounds, and the balance of sums constrains the reaction. A component can play roles in different episodes. The related metric that can be represented by numbers and thus different kinds of equations constrain the episodes. In our formulation, a component is an abstract feature, and it has a metric. The metric can be discrete, as in the case of chlorine, or continuous. We shall use 
Colors of Autism Spectrum:

this definition in the context of place cells, also called place fields, for example (O'Keefe and Nadel, 1978) that make an approximate discretization of the spatial component as well as the related grid cell system (Rowland et al., 2016) that compute the related metric. Together, these components can comprise a predictive deterministic model for cognition, e.g., if the component is represented in the neuronal substrate and, e.g., if it is equipped with attractor dynamics.

We model the representation that discretizes a component and the dynamic system that computes the metric with a predictive autoencoder. In this setting, place fields comprise the hidden representation, and grid cells contribute to the computation of the estimated future inputs given past ones. The concept of autoencoders will help us to shed light onto the enigmatic problem of making sense.

\subsection{Making sense with autoencoders}

We will use concepts developed in artificial neural networks as our tools for understanding autism. The first step is to examine how concepts related to autism may emerge in these structures. We start from the Weak Central Coherence (WCC) theory(Frith and Happé, 1994; Happé and Frith, 2006).

According to the WCC theory of autism, people with ASD are impaired in making sense. We may suspect what this claim means but without an algorithmic and thus computerizable definition, the claim is not amenable to modeling and is blurred, more so since the algorithmic process that models making sense has been philosophically enigmatic. Searle has asked two related questions:

Q1 ... If we are to suppose that the brain is a digital computer, we are still faced with the question 'And who is the user?' (Searle, 2013)

Q2 The brain is working with internal representations, but internal representation in any information processing system is meaningless without an interpreter. The homunculus paradox claims that all levels of abstraction require at least one further level containing the corresponding interpreter. The interpretation - according to the fallacy - is just a new transformation, and we are trapped in an endless recursion (Searle, 1992).

Fortunately, the fallacy can be solved by rephrasing the sentence in interpretation and turning the fallacy upside down (Lőrincz, Szatmáry, and Szirtes, 2002; Lórincz et al., 2002). Searle said that the representation of the input should make sense. The rephrased version is this: (not the internal representation but) the input, e.g., the retinal pattern, or its transformed forms, should make sense. We say that the input makes sense if it can be derived or generated internally, i.e., by means of the internal representation. According to this approach, the internal representation interprets the input by (re-)constructing it. In turn, we have a resolution to the fallacy and an architecture, the autoencoder made of the input, the representation, and the generated (estimated) input. We consider autoencoders in the context of making sense or central coherence. The transformation of one representation to another is called mapping.

Mapping connects or associates two representations, like connection between the curved surface representation of the Earth and a two-dimensional flat representation. The autoencoder is made of (at least) two types of mappings. The first maps the input of the representation. The other one maps the representation and produces an estimation of the input. Both mappings are to be learned. The representation makes sense at a given time if the input it generates closely resembles the actual input at the time of the comparison. A relevant condition in this is that delays in processing should be counteracted; we require that the input that produces the representation and the estimated input generated from the representation should match each other at any given time, despite the time required by both mappings. This constraint leads to the need for predictive mapping.

In sum, making sense requires an architecture that (i) maps the input to the representation and (ii) propagates the representation in time into the future (iii) in order to match the representation-based estimation of the input to the actual input in spite of the delays. Thus, sense-making can be corrupted in at least four ways:

(1) if the input-to-representation map, a.k.a. encoding or bottom-up processing, is erroneous

(2) if propagation into the future, a.k.a. the predictive system or recurrent collateral-based temporal encoding or model, is erroneous

(3) if the representation-to-input map, a.k.a., decoding or top-down modulation, is erroneous

(4) if action generation based on the representation that makes sense is erroneous because the final error between the input-to-estimated input is not an error of a single autoencoder embedded deeply into parallel-distributed processing but is an error between the planned action and the action that occurs and is observed. This loop of observation, computations and action launching has considerable delays that must be counteracted by predictive autoencoders.

There is a tacit assumption here, namely, that sensemaking involves conflict resolution, since conflicts may arise between parts or components of the representation. Thus, we assume the presence of components and the previous and ongoing learning of them. We assume that representations and estimations of the diverse components are imprecise, but decision-making requires the resolution of conflicts between them. In turn, we have a fifth and sixth possibility for potential impairments:

(5) if component formation is imprecise or corrupted 
Colors of Autism Spectrum:

(6) if timely conflict resolution is suboptimal or impaired.

All the underlying neural mechanisms may be corrupted and may contribute to ASD. For example, an error may occur if component formation concerns the binding of multi-modal sensory inputs or if model construction and prediction, and thus temporal propagation, in the internal, possibly attractor network is imprecise.

\subsection{The neural system of component learning}

Any component corresponds to an abstraction as opposed to sensory information. Such abstractions can simplify cognitive tasks provided that the relevant components are selected for solving the problem. A component is of high value if helps to decrease the number of required components. An example is a coordinate system that is tightly aligned to the environment, a.k.a., laboratory coordinate system or allothetic system, the counterpart of the egocentric or idiothetic system.

\subsubsection{The discretization of the allothetic space component}

Place fields are neurons that respond to overlapping but small local regions in the available space, and their responses in that region are almost independent from light conditions, head directions, body position, and gaze direction, among other factors. In turn, they discretize the available space and strip off peculiarities of the individual fields. The discretized space is typically two for rats (O'Keefe and Burgess, 1996) and can be three for bats (Yartsev and Ulanovsky, 2013). In turn, place fields and the corresponding metric form a component.

Place field representations occur in the hippocampus, one of the main parts of the entorhinal-hippocampal complex (EHC). The entorhinal cortex contains grid cells and head-direction cells that, together with the place cells, can give rise to an implicit representation of the metric (Moser, Moser, and McNaughton, 2017). Many details of the EHC structure are known, and the structure encompasses almost all problems that, in diverse combinations, may lead to ASD and may also occur in and between neocortical areas. Below, we describe the EHC, which forms a loop. It has been interpreted as an autoencoder (Lőrincz and Buzsáki, 2000; Chrobak, Lőrincz, and Buzsáki, 2000).

\subsubsection{The loop}

The hippocampus is part of the loop. It is a relatively small archicortical structure at the top of the sensory processing pathways. Some of its inputs arrive from different sensory modalities through the neocortex to the superficial layers of the entorhinal cortex (EC).
The efferents of these layers target different substructures of the hippocampus, such as the dentate gyrus (DG), the CA3 and the CA1 subfield. The output of the hippocampus is the input of the deep layers of the EC. Efferents of the EC's deep layers close the loop by targeting the superficial layers of the EC. Efferents also spread throughout the neocortex, possibly to close larger loops in a direct or indirect manner. For a depiction of the anatomy of the hippocampus and its pathologic conditions, see (Dekeyzer et al., 2017). For more details on the evolution of this structure and on its environment, see (Witter, Kleven, and Flatmoen, 2017) and (Witter et al., 2014), respectively.

Grid cells appear in the medial entorhinal cortex and form a triangular attractor network that precisely measures any changes in position. For a review of grid cells, see (Rowland et al., 2016). There are two types of these grids. The responses of one of them are head direction independent. Other neurons exhibiting triangular activities are sensitive to head directions. The interdependence of grid cells and place cells is complex; they assume each others' roles, and spatial representation becomes corrupted (in diverse ways) if any of the constituents of the loop is lesioned, even if the deactivation is only transient.

Head direction cells (Taube, Muller, and Ranck, 1990) are present in different brain structures, including the entorhinal cortex (Taube, 2007). They are necessary for the formation of both place cells and grid cells (Taube, 2007). This specific feature was exploited as a semi-supervisory signal in the deep learning model that developed both place cells and grid cells from visual and head direction information (Lörincz and Sárkány, 2017).

The responses of place fields are useful high-level abstractions, justifying the use of the name 'cognitive map' for the hippocampal CA1 and CA3 subfields where these neurons reside. Discretization of the allothetic coordinate system alleviates the curse of dimensionality and makes path planning (exponentially) easy.

\subsubsection{Episodic memory from temporal relations}

It is generally believed that declarative memories depend on the EHC loop (Buzsáki and Moser, 2013). Episodic memory encodes temporal sequences and can replay them. Replays are launched in the CA3 subfield of the hippocampus via its large recurrent collateral network system. Replays have a very specific waveform and create the most synchronous known population pattern in the brain (Buzsáki, 2015). They occur during consummatory behavior and non-REM sleep. Their particular waveform is called sharp waves (SPWs) and ripples or SPW ripple complexes. SPWs have large amplitudes and a negative polarity. They are 40-100 ms long. In many cases, they are accompanied by ripple waves of $110-200 \mathrm{~Hz}$.

SPW ripple complexes are necessary for memory consolidation and the transfer of memory to neocortical 
Colors of Autism Spectrum:

areas. They have high excitatory gains for efficient transfer, and these high gains may give rise to epileptogenic episodes; indeed, the hippocampus is the most prominent source of epileptic high-frequency oscillations (Bragin et al., 1999).

\subsubsection{Forming semantic memory by factoring out time}

In addition to temporal sequences or episodes, semantic memory is the other type of explicit memory formed by the complex around the hippocampus. Our view on semantic memory is very similar to the view described in (Buzsáki and Moser, 2013). The putative 'algorithm' is widely used in natural language processing and image processing, where it is called 'bag of words' and 'bag of visual features' representation, respectively. We describe the former. Documents contain many words organized by time according to syntactic rules. The category of the document can be classified quite accurately by means of the number of occurrences of the words in the document upon normalization. Many words will have zero occurrences, but contextually important words will have high values. Time and thus syntax are neglected in this representation, and only the cooccurrences are retained. This concept is similar to the notion of 'recognition-by-components' (Biederman, 1987), in which a structure, e.g., a face, is recognized as a face by the components it has, sometimes even in the presence of syntactic errors. In turn, constituents form one part of semantic memory. In addition, the meaning of a word can be disambiguated by the list of other words surrounding it, see (Pintér et al., 2015) and the references therein. The difference between the set of constituents without and with the context, in the case of partial observation, is illustrated in Fig. 2. Thus, we define the semantic memory belonging to an entity as the time-independent collection of its constituents and the context of which that the entity is a constituent. Semantic memory forms an associative structure that is robust against timing requirements.
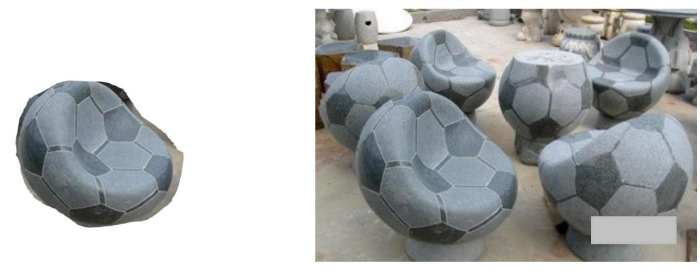

Figure 2: Components and context dependence Spatio-temporal context can modify the interpretation. (a) Interpretation of the structure defined by its components: A soccer ball that needs inflating. (b): Interpretation of the same structure in its context: A chair.

We reinforce the view that time compression is a key to understanding the time-independent nature of semantic memory that leads to efficient bag-like rep- resentations (Lőrincz and Sárkány, 2017). We quickly review why we adopt this view.

In rats, spike sequences during theta cycles are timecompressed versions of the place fields belonging to the rat's trajectory (Dragoi and Buzsáki, 2006; Lisman and Jensen, 2013). This compression in the entorhinalhippocampal loop is the result of theta phase modulation of gamma wave power; see, e.g., (Buzsáki and Moser, 2013) and the references therein. Theta oscillations are in the $6-10 \mathrm{~Hz}$ interval, whereas the frequency of gamma oscillations is between $30-120 \mathrm{~Hz}$. The time constant of the neurons receiving these modulated signals is between $10-30 \mathrm{~ms}$, and thus, synapses formed with those neurons can send spikes during this relatively long interval. Since cell assemblies of the hippocampus have a lifetime of approximately the same duration (10-30 ms), neuronal assemblies that fire within this time window will be learned by the downstream neurons receiving their signals without any information about their temporal order. As it happens, neurons responding to a trajectory of approximately $1 \mathrm{~s}$ travel time fire within that $10-30 \mathrm{~ms}$ time window. Context is also available for associative learning; since the positive cycle of the theta wave is approximately $100 \mathrm{~ms}$, it has a number of gamma oscillations, and the time they can cover in $100 \mathrm{~ms}$ is on the order of approximately $2 \mathrm{~s}$.

\subsection{Consciousness and component binding}

Components may be observed through different modalities. Such signals propagate through different channels and can have different delays. Delays in a synchronized autoencoder are compensated by predictive structures, and consequently, delays in the different processing channels can be matched. This is a sophisticated multipurpose algorithmic structure in the neuronal architecture, and each of the elements may be corrupted, disrupting cognition.

There are multiple options for interpretation, but only one may survive at a time, and selection seems occur unconsciously. This problem of unconscious selection and disambiguation was noted by von Helmholtz approximately 150 years ago. He based his arguments on multistable visual perception, demonstrating that under certain conditions, there should be only a single interpretation at a time.

Due to the different delays in the different processing channels, the single - typically time-dependent interpretation cannot change quickly but should be maintained for at least the duration of the cumulative delays in input-output processing, i.e., for delays between the inputs of sensory channels and the motor outputs. Such constraints are plausible since the world is deterministic. Experimental findings support our view: Switching in multistable perception binocular rivalry experiments can take as long as $1 \mathrm{~s}$ (Leopold 
and Logothetis, 1996; Brascamp et al., 2005). For a recent review, see (Brascamp et al., 2018).

In the case of binocular rivalry, the single interpretation of the interpreting autoencoder is 'in error', since the other potential interpretation is diminished until perception switches. Such mistakes cannot be avoided if a single interpretation is required. Imprecise delay compensation, however, can lead to different errors that may give rise to hallucinations. We shall consider such issues later.

According to theoretical predictions (Lőrincz and Buzsáki, 2000; Lőrincz and Szirtes, 2009), delay compensation depends on the hippocampus, which also drives the learning in neocortical structures. Lörincz and Buzsáki suggested that delay lines in the loops formed by the dentate gyrus and CA3 serve the adaptation process needed to compensate for the signals within the hippocampus and those that arrive from the neocortex, including the entorhinal cortex. The assumption is partially supported by the experiments of Henze et al. (2002). They found tunable and long delays in this loop. More recent results provide further support: Khodagholy et al. (2017) monitored multiple sites in the rat neocortex and found that (i) ripples in the association cortex were similar to hippocampal ripples, (ii) these ripples were coupled and (iii) coupling was strengthened during sleep, suggesting that delays were compensated for dynamically.

We consider the binding of components a 'synonym' for the learning of spatial and possibly temporal contexts, or both, that can be multi-modal and may be subject to delays. The context learning depends on REM sleep, as indicated by the REM-specific optogenetic silencing of GABAergic neurons of the medial septum projecting to the hippocampus in mice, which diminished learning (Boyce et al., 2016).

\subsection{Two-stage operation: Memory en- coding and consolidation}

Further complications arise from the need to encode memories, transfer them to neocortical areas and consolidate them. There are two stages of explicit memory: Experiencing and consolidation. They occur separately, as first suggested by Buzsáki (1989). He suggested that during exploration, i.e., during the theta waves, neocortical information enters the hippocampus and induces weak and transient heterosynaptic potentiation in the CA3 subfield. Later, these weakly potentiated neurons and neuronal chains cause sharp waves during consummatory behaviors that give rise to long-term synaptic modifications in the hippocampus. This model has gained considerable support over time on both theoretical (Hinton et al., 1995) and experimental grounds. Recent experimental support is reviewed below.

\subsubsection{Brain waves contribute to memory consol- idation in many ways}

Due to spike time-dependent plasticity, proper timing of neuronal spikes is critical for long-term potentiation and the long-term depression of synaptic strengths. There are stringent requirements of memory learning and consolidation, since (i) they require two stages, the exploratory and the consummatory stages, and (ii) there are two types of memory formation tasks, namely, the learning of temporal series and the learning of semantic relations.

Theta waves are present in the exploratory stage. Their presence poses considerable challenges since time is needed for both learning temporal series and temporal compression for learning semantic relations. The process is controlled by the two efferents of the EC entering the hippocampus: Neurons of layer 3 of the EC innervate the CA1 subfield, whereas efferents of layer 2 entorhinal neurons enter the CA3 subfield. The interplay between the two channels controls spike timing and the coupling between theta and gamma waves (Fernández-Ruiz et al., 2017).

The consummatory stage is also complex since memory consolidation should involve both the EHC loop and remote sites in the neocortex. Sharp waves (SPWs) play an important role in these processes. However, SPWs are not the only player; other brain waves contribute to this process, but the full machinery is not yet known. Some details follow below.

According to the experiments, slow-wave sleep (SWS) seems to be more involved than REM sleep in the consolidation of the system, but both types of sleep contribute (Diekelmann and Born, 2010; Born and Wilhelm, 2012). SWS or non-REM sleep is characterized by joint spindle and ripple events. During these events, the depolarizing up phases of slow oscillations reactivate the hippocampal time series and SPW ripples. Different brain wave frequencies can couple, and slow waves seem to restrict the time interval for coupling when faster oscillation occurs within slower ones, a phenomenon called phase biasing. In turn, slow oscillations can orchestrate local processes globally, making SPW ripples the most synchronous wave pattern in the brain. Another observation is that these ripples can stabilize the place cells in the hippocampus (Roux et al., 2017).

However, Boyce et al. (2016) showed causal evidence that contextual memory consolidation depends on theta rhythms during REM sleep: they developed a REM sleep-specific optogenetic silencing method for medium septal GABAergic neurons and showed that silencing during REM sleep impairs the formation of fear-conditioned contextual memory. In this regard it may be worth mentioning that according to simulations (Lőrincz and Sárkány, 2017) both temporal and semantic memories can be important for component formation accompanied by the development of the related metric, important elements of cognition. 


\subsubsection{Consolidation of emotional components}

The consolidation of emotions in their respective contexts involves additional brain structures beyond the EHC and the neocortex. There is general agreement that emotions are processed by the amygdala, and anatomy supports this additional structural requirement:

(i) the ventral hippocampus projects directly to the basolateral amygdala and the central amygdala, and connections are reciprocal (Pitkänen et al., 2000),

(ii) fear can be switched on and off in distinct circuits between the amygdala, hippocampus and the medial prefrontal cortex by selective activation of specific neuronal circuits (Herry et al., 2008), and

(iii) multiple parallel pathways exist between the amygdala and the hippocampus (Xu et al., 2016). One pathway encodes the context-dependent retrieval of cued fear memories. Another pathway is concerned with fear behavior in a contextdependent manner.

In turn, fear is an internal component that characterizes both episodic and semantic memories.

Experimental studies on the consolidation of emotional components have been published recently (Girardeau, Inema, and Buzsáki, 2017). Their findings that reactivations of memory traces in the basolateral amygdala peaked during hippocampal SPW ripples are in line with other consolidation patterns.

\subsection{Bottom-up and top-down process- ing serves component formation}

The conditions for the learning of components are complex and are listed below:

C1) The representation of any component is to be made free from other components. For example, shape may not involve color, and form should be separated from motion. However, behavior is based on the different behaviorally relevant components, and the interpretation of these components depends on the context; thus, they require associations between the separated components. Such associations between components look similar to multi-modal information fusion, with information fusion providing the separation and the pattern completion capabilities between the components and within the components, respectively. Figure 2 serves illustrative purposes in this respect.

C2) Components - in the ideal case - come in two different forms. Episodic components address autobiographic memories and are restricted in space and time. Semantic components differ; they are non-episodic and represent a combination of components, are influenced by the context and are independent of time.
C3) Component formation is to be accompanied by the development of a metric for the components to enable model constructions, predictions and 'path' planning.

C4) Component formation requires the synchronization of inputs with the representation and the representation-based estimation of the inputs. This triad forms a loop made of (i) bottom-up (downstream) processing, (ii) predictive (recurrent collateral based) processing, and (iii) topdown (upstream) estimation. The loop enables model construction in the representation, tuning and synchronization given the error between input and estimated inputs; see, e.g., (Lőrincz and Szirtes, 2009) and the cited references therein.

Now, we turn to constraints on the network level. These constraints emerge from considerations regarding neural firing and synaptic energy consumptions, wiring costs and, last but not least, the characteristics of natural signals.

\subsection{Sparse representations}

Neural computation is 'expensive'; the brain consumes considerable energy. There are two main contributors to energy consumption: Neuronal spikes and synapses. Fifty percent of the energy is used on postsynaptic glutamate receptors, $21 \%$ on action potentials, and $20 \%$ on resting potentials; energy is also spent on presynaptic transmitter release and transmitter recycling (Howarth, Gleeson, and Attwell, 2012; Harris, Jolivet, and Attwell, 2012). Energy insufficiency may involve several neuropathologies.

\subsubsection{Theoretical considerations regarding spar- sity}

Given the considerable costs of neuronal resting potential, action potential and postsynaptic receptors, the neural system should minimize firing. Connections require volume, and propagation along the axons and dendrites takes time, giving rise to limitations in the size of the brain. In turn, connectivity should also be sparse. How can such constraints support cognition? Sparse representations seem to be related to the characteristics of the statistics of natural signals. Such statistics have a general feature; they exhibit power law behavior (see, e.g., the work of Ruderman (1994) on images and that of Lewicki (2002) on sounds as well as the references they cite). Neuronal nonlinearities are well adapted for such signals (Schwartz and Simoncelli, 2001), and the proper compression method for such signals with heavy-tailed distributions is sparse representation (Olshausen and Field, 1996), especially since it meets the constraints on energy consumption.

Furthermore, searches for optimal components of the sparse representation are generally subject to combinatorial explosion, but it is not a necessity for com- 
ponents assuming heavy-tailed distributions due to a good approximation called L1-Magic (Candès and Romberg, 2005). Furthermore, learning in deep sparse artificial neural networks has favorable mathematical convergence properties (Arora et al., 2014), suggesting that evolution found a tractable solution for component learning and exploitation based on the data structure that fits cognition with limited memory span constraints. In addition, the neuronal structure and dynamics are well adapted to power law distributions and to related scale-free small-world structure and, on the modeling side, to representing the dynamic processes of such structures; see, e.g., (Singer, 2013; Cocchi et al., 2017) and the references therein.

\subsubsection{Constraints and errors in sparse represen- tations}

In a typical network with sparse representation, the input excites all neurons downstream first. Then, the generative process generates an estimated input, as in the model of Olshausen and Field (1996). The difference between the input and the estimated input is used to correct and sparsify the representation, and the interaction continues in a second generative step and then further on. Thus, in addition to consuming high amounts of energy consumption, forming the representation is time consuming. Sparse representation can take advantage of the generative step, and the encoded memories are similar to those found in the brain (Olshausen and Field, 1996; Lewicki, 2002).

There are different solutions to the abovementioned problems. For example, one may use sparse representation for the supervisory training of a deep neural network. The result will be much faster (Sprechmann, Bronstein, and Sapiro, 2015), and the solution has the promise of large gains in energy consumption. The drawback is that many layers may be needed.

It seems, however, that neuroscience has found other solutions and uses fast feedforward and feedback competitive inhibitions; nature 'developed' a large set of different interneurons. Neural representation in the hippocampus is highly sparsified, and inhibitory circuits form a sophisticated network that incorporates a considerable number of different interneurons (Freund and Buzsáki, 1996). One may suspect that it is hard to balance the excitation/inhibition ratio in the different stages of exploratory and consummatory behaviors and the consolidation processes of episodic and semantic memories.

Another solution for saving energy and gaining speed is to group similar neurons and set up competition among them. This is the so-called structured sparse representation (Bach, 2010). This structure offers fast feedforward estimation of the groups that are to be selected (Lőrincz et al., 2016). Lőrincz et al. (2016) considered structured sparse representation as a model of minicolumnar organization. This minicolumnar organization is another potential source of problems in autism. We shall return to this point later.

GABA concentrations correlate with the efficiency of the suppression of neural activities, although in a nonuniform way, as they are dependent on the ongoing cognitive tasks. For example, in the case of voluntarily suppressing memory retrieval, (i) reduced hippocampal activity and reduced memory for suppressed content was found, (ii) greater resting concentrations of hippocampal GABA predicted better mnemonic control, and (iii) higher hippocampal GABA predicted stronger fronto-hippocampal coupling during suppression, whereas (iv) the level of prefrontal GABA did not influence the effect (Schmitz et al., 2017). We conclude that voluntary control and thus the manipulation of unwanted components require greater GABAergic influence.

As previously mentioned, coupling and coherence between brain waves seem critical for memory formation and consolidation. Recently, Ognjanovski et al. ((Ognjanovski et al., 2017)) studied single-trial contextual fear conditioning. They found that network oscillations in the CA1 subfield showed greater firing coherence with fast-spiking interneurons. Fast- spiking interneurons are GABAergic interneurons that most likely contain parvalbumin. The inhibition of parvalbumin -expressing interneurons blocked the consolidation of fear memory. Blocking also involved losses in delta, theta and ripple waves and CA1 network coherence. However, when parvalbumin interneurons were activated rhythmically, network coherence improved.

The interplay between neuromodulatory constituents and neurotransmitters is also complex and delicate. Different molecules may act and balance each other in very specific ways. For example, deletion of neuroglinin-3, a postsynaptic cell adhesion molecule (Polepalli et al., 2017), decreases NMDA receptormediated postsynaptic currents and increases the probability of presynaptic glutamate release in parvalbumin interneurons. The net outcome of the changes is that gamma oscillations and sharp wave ripples are reduced, and the extinction of contextual fear memories is decreased, whereas fear conditioning does not change.

Since the energy demand of fast network oscillations is high (Kann, 2012) and since parvalbumin-containing basket cells make approximately $50 \%$ of the interneurons capable of generating such oscillations, one expects that an energy deficit of parvalbumin neurons leads to diverse impairments in functioning, a finding supported by recent experiments (Inan et al., 2016).

\subsubsection{Autoencoding and interpretability}

As previously noted, sparse representation is formed by the autoencoding principle. This is so since sparsification of the representation alone is fragile and may give rise to low quality or possibly zero activities, whereas the autoencoding principle forces the network to avoid such trivial representations. The quality of the representation can be maintained if the representation is 
Colors of Autism Spectrum:

supposed to save all or most of the information about the input, i.e., if it can generate a high-quality estimation of the input. We shall call the difference between the input and the predictive estimation of the input reconstruction error. Sparse representation compresses the input if the number of active (spiking) neurons in the representation is smaller than the number of active units in the input. This is called sparse compression. Similarly, L1-Magic is also called compressed sensing to emphasize this particular property; see, e.g., (Donoho, 2006) and the cited references.

Sparse compression differs from general data compression methods, such as the Zempel-Liv algorithm in PKZip, Gzip and png, since it must regenerate the input using only a few active neurons. Component learning, then, is a natural tendency of this method; it is often called sparse dictionary learning (Rubinstein, Zibulevsky, and Elad, 2010) because of the analogy that although a dictionary contains many components, a few components can make a sentence. The better the autoencoding property and the sparser the representation, the lower the energy consumption and the better the dictionary, provided that information is spared.

\subsubsection{Impairments in the neuronal networks}

Since we assume that (i) impairments in social behavior are the result of corrupted component formation and exploitation and (ii) component formation modifies brain connectivity, there are at least two associated falsifying issues:

1. neuronal development is atypical in autism and impairs network modifications and thus component formation,

2. neuronal activities are atypical, and the activity patterns impair the exploitation of components.

Such differences can lead to different types of autism and different comorbidities. We review brain structure impairments below.

\subsubsection{Brain structure impairments}

Atypical neuronal connectivity structure means atypical development. This atypical development might occur at different times, e.g., during early development or later, and both are general features of autism. Larger brain sizes have been noted in individuals with ASD, and Hazlett et al. (2017) suggests that the atypical increase in brain volume occurs early during development. They found that surface area information form magnetic resonance images of the brains of 6 to-12-month-old children has a high power to predict whether the child will later be diagnosed with autism.

Larger surface areas of the same volume involve different gyrification patterns, as have been reported in the literature for the left pre- and post-central gyrus (Ecker et al., 2016). Increased gyrification seem to enable an increase in the number of short-distance connections; see, e.g., (Courchesne and Pierce, 2005), for a review.

The minicolumnar organization of superficial layers 2 and 3 of the neocortex is not yet understood, and it has been debated whether it has a function at all or it is only a consequence of the distribution of doublebouquet cells in those superficial layers (Horton and Adams, 2005). There is significant variation in distributions in different areas, including the portion of the ratios of the two subtypes of these double-bouquet neurons (Yáñez et al., 2005). The same paper also reports that these GABAergic interneurons are not present in rodents, lagomorphs, and artiodactyls and are present but in relatively low numbers in carnivores. There is a lack of consensus regarding the size, structure and interaction of the minicolumnar structure and the related changes during development.

According to some works, the minicolumnar structure is reduced in autism; the total number of neurons in individual minicolumns seems normal, but the distance between minicolumns appears to be reduced (Casanova et al., 2006). Other works present different results. For example, McKavanagh et al. (2015) report that wider minicolumns are more pronounced in younger ages in ASD. The origin of the discrepancy is unclear. Page et al. (2018) report that a gene associated with both schizophrenia and autism regulates the columnar distribution in the prefrontal cortex, and this regulation is activity dependent. Experimental results indicate that environmental effects and potential differences in the cohorts of autistic individuals may account for the discrepancies. Developmental trajectories may indeed differ, as reported by Tek et al. (2014) and as indicated by studies of discordant monozygotic twins (Willfors et al., 2017). A detailed discussion of the different findings regarding organization can be found in (Dickinson, Jones, and Milne, 2016). They consider the diversity of abnormalities associated with ASD and the number of individuals included in the experiments.

\subsubsection{Differences in axial diffusivity, myelina- tion, and synaptic homeostasis}

Ecker et al. (2016) also found that increased gyrification is accompanied by atypical neural axial sprouting, which is most pronounced in axons traveling close to the cortical sheet. According to their report, enhanced gyrification correlates with increased axial diffusivity in general, and the relationship may be causal in either direction. Such changes may influence the non-linear dynamics of the brain, which execute highly interconnected parallel and serial operations close to the socalled critical region characterized by spatio-temporal avalanche dynamics; these dynamics are heavy tailed, have close to a $1 / \mathrm{f}$ power distribution, the power of brain oscillations is inversely proportional to the frequency of the oscillations, as is typical in scale-free small world structures (Buzsáki and Draguhn, 2004; Singer, 2013). Changes in intermediate structures 
Colors of Autism Spectrum:

can form bottlenecks in information processing and information sharing, including the formation of new representations.

Structural differences can influence information propagation and synchronization in the neuronal substrate. An additional parameter is myelination. According to Buzsáki et al. (2013), axon size and myelination seem to be the most important factors for the scaling of network oscillations because they determine the conduction velocity of neurons. One may suspect changes in the scale-free network property due to changes in axial diffusivity within local regions, especially if myelination also changes, as described in (Ecker et al., 2016). Furthermore, such local changes may influence the sensitivity and variations of local neural responses, an intriguing feature in autism (David et al., 2016b).

Synaptogenesis is an additional critical matter. Changes in spine morphology can be vital in forming large networks, and troubled synaptic connections are considered the main underlying reason for autism by many researchers (Toro et al., 2010; Penzes et al., 2011). According to the data, increased spine density gives rise to decreases in cognitive functioning in ASD, supporting the view that ASD can be characterized by denser connectivities locally and hypoconnectivity globally (Hughes, 2007).

\subsection{Summary of some phenomena that may impair component learning and cognitive manipulations}

We have collected some factors that may influence component learning and manipulation, but the list is not exhaustive. The starting point is the autoencoder model (Lőrincz and Buzsáki, 2000; Lőrincz and Sárkány, 2017). The autoencoder is a comparator that computes the difference between input and the estimated input, the latter of which is derived from the representation. Comparison requires compensation for the processing delays in the loop. Delay compensation can be accomplished by prediction. Imprecise prediction, imprecise timing, and imprecise spike timedependent plasticity can impair component formation.

Components have low dimensions that enable discretization of the space of the component, as in the case of place cells that form the 'cognitive map'. Discretization is made possible by means of highly sparse, possibly almost winner-takes-all representations. A lack of sparsity corrupts component formation.

To control the components - e.g., to navigate in allothetic coordinates - a representation of the metric is necessary to estimate the magnitude and the direction of control outputs. Particular forms of metric learning exploit the interplay between semantic and episodic memories; see, e.g., (Dragoi and Buzsáki, 2006; Buzsáki and Moser, 2013; Lőrincz and Sárkány, 2017) and the references cited therein. Imprecise interplay including imprecise timing can corrupt component formation and the learning of precise models.

Time compression seems necessary for semantic representation, and it can factor time out due to the temporal features of $\mathrm{Ca} 2+$ channels, which stay open for a few tenths of milliseconds. Factoring out becomes possible by averaging related temporally compressed episodes during the few tenths of milliseconds, the time that adapting synapses are open. Impairment in the durations can corrupt component formation in two ways. If the temporal window is too short, then averaging can be corrupted. If it is too long, then overgeneralization may occur.

Episodic memory can be replayed in time, which makes it similar to a predictive model; however, it needs no input. Proper temporal ordering and replaying capabilities are needed for this memory type. Memories are organized in a topographic manner in the neocortex, similar components are close to one another and mutually inhibition is typical. In the absence of such local arrangement, accidental simultaneous firing may lead to false associations via Hebbian learning. Too many spikes, e.g., the noisy brain, may lead to similar unwanted associative connections. The lack of localization or excessive axial diffusivity may also impair component learning.

There are general processes that may corrupt component learning, such as synapse formation, strong recurrent brain waves (i.e., seizures that can destroy learned information), temporal discrepancies due to insufficient myelination, improper synchronization of brain waves (e.g., theta and gamma waves during exploration) and improper synchronization of slower waves during synaptic strength consolidation (e.g., during sleep).

The learning of components serves component manipulation, such as cognitive control and motion control. Control actions should be deterministic and require temporal coordination based on perception. In turn, perception should be unambiguous, and a single, dynamic interpretation is required always despite the delays in both different sensory processing channels and action execution. The interpretation should explain (estimate) the input in all modalities and with high fidelity time estimations. In cases with more than one potential interpretation, these interpretations should satisfy the constraints of the deterministic world; while only one interpretation may be present at a time, interpretations with similar probabilities should be able to switch.

The list of the constraints posed by component formation and exploitation is as follows:

(i) intact bottom-up processing with sparsification, which requires feedforward and feedback inhibitions, as illustrated in Fig. 3,

(ii) intact top-down processing for training long-term memories, which requires predictive model-based synchronization (Fig. 3),

(iii) predictive models for forward propagation in time, 
which require recurrent collaterals (Fig. 3),

(iv) learning of delays and compensation of delays for multi-modal fusion,

(v) intact gamma and theta waves for time compression, both for semantic memory and for encoding temporal sequences,

(vi) intact slow waves, spindle waves and sharp waves for consolidation and for the transfer of learned memories to the neocortex,

(vii) unconscious selection of a single interpretation for conscious observation,

(viii) unconscious synchronization of conscious observation of the perception-action loop for precise action planning, launching, and plan execution,

(ix) unconscious switching between alternative interpretations, but not sooner than the integrated sum of the delays and synchronization time in both the sensory processing and action execution pathways,

(x) discretization of the learned separated components in the same manner as place cells,

(xi) separate and synchronized prediction of the learned components, as illustrated in Fig. 3(b), and

(xii) modulation of inputs at will, i.e., the focus of the attention process needed to decrease the combinatorial variations to disambiguate the representation within the context of the actual task.

Component learning and component exploitation are many-faceted mental processes. We believe that any of the listed points may be corrupted and may impair these processes. These corruptions have different origins and can be cumulative, impairing the handling of high-complexity cognitive problems first, partially due to the lack of components that could simplify the task and partially due to the impaired manipulation of the existing components, including control actions.

We argue that social behavior is one of the most involved tasks since it concerns at least one individual other than the self, which increases the number of variables, including the number of hidden variables and has time constraints in analyzing, making decisions and controlling the situations. Consequently, we view autism as the tip of the iceberg of the cumulating problems of component formation and exploitation.

In the following, we shall review comorbidities and try to pinpoint to their underlying causes. Afterward, we review the theories developed to explain autism and their strengths and weaknesses. We will then relate these theories to our model, which relies on the complexity of component formation and exploitation.

\section{3 'Results': Falsifying issues}

The very first observation is that the list of potential impairments that may contribute to autism - that is, to a 'single' behavioral problem - is extremely broad and varied. ASD has an exclusively behavioral definition, and research has uncovered tremendous and divergent potential causes. The behavioral characteristics include the early onset of difficulties interacting with others, which in many cases are accompanied by restricted interests and repetitive activities. The large set of potential causes is accompanied by a high probability of comorbid traits with different behavioral characteristics. How can it be that a problem with social behavior may co-occur with impulsive ADHD, epileptic seizures, hallucinations, and synesthesia as well as problems with goal-directed actions?

We have previously proposed that the common reason is the formation and exploitation of components. Components are complex and uncertain, especially when they are hidden. Nonetheless, the selection of the smallest number of relevant components is critical in decision making. This is the case in social interactions, in which estimations of mood, feelings, level of knowledge and capabilities are necessary for the success of joint activities. In the previous section, we listed the neural processes needed for the learning and manipulation of components. In this section, we consider the underlying reasons for the comorbidity of different disorders. We consider justifications or reasonings regarding comorbidities one of the strengths of our model, especially since this is the weak point of most models. We shall consider other models in the Discussion.

We start with an observation that was thoroughly detailed by Buzsáki, Logothetis and Singer (2013) and was mentioned previously: During the evolution of the mammalian brain, brain wave frequencies and their linear spacing on a natural logarithmic scale from $0.03 \mathrm{~Hz}$ to $300 \mathrm{~Hz}$ remained approximately the same. Coordinated activities, at least for a subset of neuronal activities across the whole brain, seem critical despite the relative sizes of different areas and the enormous growth of the whole brain.

\subsection{Comorbid disorders}

Here we review some typical comorbid disorders.

\subsubsection{Epilepsy}

Epilepsy is a neurological condition. It is often comorbid with other neurologic and psychiatric disorders. Clinical overlap with ASD is high, and there are many comorbid profiles. The prevalences of epilepsy in males and females are approximately $18 \%$ and $34 \%$, respectively (Amiet et al., 2008). There are many candidate genes that could be responsible for the comorbidity; while they are mostly involved in the formation, remodeling and maintenance of synapses and neurotransmission, there are also other dependencies (Lo-Castro and Curatolo, 2014).

Epilepsy is considered a network problem (Kanner, 1943). As such, it is fragile in many ways, including 
(a) Bag Model

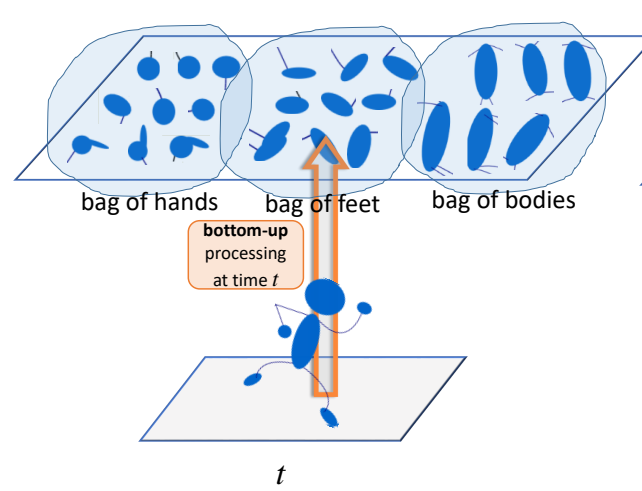

(b)

\section{Spatio-Temporal Model}

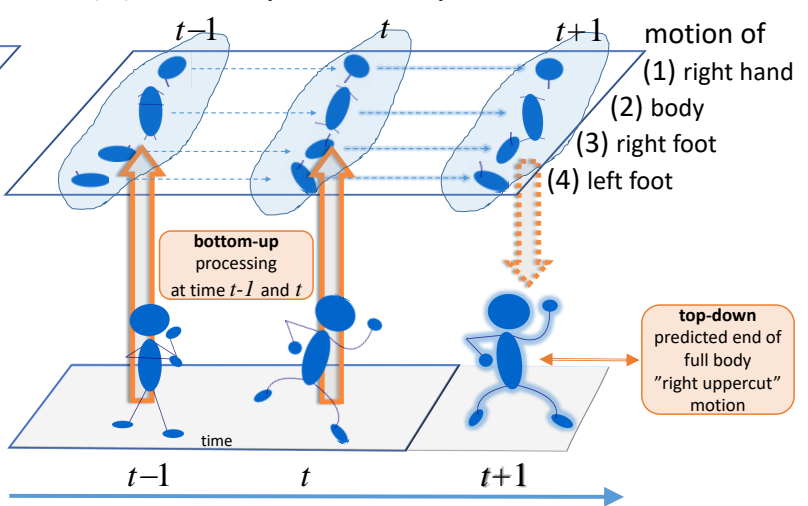

Figure 3: Autoencoding principles.

There are two model types, depicted in (a) and (b). The figures serve illustrative purposes. Notations: lower level represents the input. Upper level shows the representation. Body parts at the level of representation (light blue clusters) represent indices, or neurons in the representation and the corresponding top-down vectors that should reconstruct the input and are excited via bottom-up processing. (a): The bag model: Input is broken down into components. Only a few elements of the representation correspond to the input, and thus, representation becomes sparse. (b) The predictive spatio-temporal model: Upon bottom-up processing, the spatio-temporal model propagates in time and predicts - with some uncertainty - the input. The estimated input can be compared with the actual input if propagation can compensate for the delays in bottom-up, top-down and model computations. Error in the delays can tune delay lines. Error in the prediction can tune the predictive model. The interested reader may wish to consult the literature cited in the paper for details of the mathematics involved. Image source: https://openclipart.org/search/?query=stick \pm man

network centrality (Balardin et al., 2015), a potentially serious bottleneck for coordinated activities. Seizures may be due to high excitation/inhibition ratios, which are also one of the main theoretical routes proposed in autism models (Rubenstein and Merzenich, 2003). In turn, a high degree of genetic overlap can be expected, and indeed, ASD and epileptic encephalopathy seem to have many common genetic causes (Srivastava and Sahin, 2017). Restricting particular features, autismepilepsy phenotypes with macrocephaly may lead to the separation of relevant subtypes and to improved genetic screening, as suggested by Marchese et al. (2014). The co-occurrence of interictal electroencephalogram abnormalities, regression, and macrocephaly was suggested by Valvo et al. as a distinct endophenotype of ASD (Valvo et al., 2016). Another connection is suggested by Bozzi et al. (2016): An excitation-inhibition ratio imbalance resulting from neurodevelopmental deficits may be the common pathogenic mechanism, and this hypothesis seems to be supported by genomewide association studies. However, according to Dickinson et al. (2016), claims regarding the net increase in excitation or inhibition in autism have little support, if any. Inflammation has also been suggested as another route for the neurobehavioral comorbidity of epilepsy and ASD (Mazarati, Lewis, and Pittman, 2017).

Importantly, there are many metabolic and regulatory pathways underlying autism, some of which seem unique to this condition (David et al., 2016a). The quest to discover causality relations, including the necessary and sufficient conditions, is difficult. However, the cited genetic study (Srivastava and Sahin, 2017) indicates that the genes involved in comorbid disorders have undergone more purifying selection than the genes unique to ASD.

Since there are many interdependencies between autism and epilepsy, it is difficult to pinpoint to any specific component-forming mechanism. An important exception is cognitive impairment, which is the most common outcome of epilepsy; epilepsy can cause considerable harm to the developing brain (Holmes, 2016). The main connection in our model is that epilepsy gives rise to morphological and physiological changes, modified synaptogenesis and altered excitatory and/or inhibitory balance, which destroy both network structure and dynamics and increase the severity of the component formation impairment. A falsifying issue is the type of impairments that may occur in mesial temporal lobe epilepsy, i.e., epilepsy at the core structure for forming the episodic and semantic memories that we consider the key to understanding autism. According to novel findings (Okruszek et al., 2017), mesial temporal lobe epilepsy gives rise to social cognitive deficits, including significant mentalizing deficits; these findings support our proposal.

Further indications come from brain waves: (i) Sharp wave ripples may undergo pathological conversions called 'p-ripples', which are the marker of epileptogenic tissue (Buzsáki, 2015); (ii) Gelinas et al. (2016)ed memory consolidation, offering a more direct route to the impairment of component formation and thus the comorbidity of ASD and epilepsy. Furthermore, the rat model of temporal lobe epilepsy shows that interictal epileptiform discharges are precisely coordinated 
with spindle oscillations in the prefrontal cortex during non-REM sleep and that similar correlations occur in subjects with focal epilepsy (Gelinas et al., 2016).

The abovementioned 'p-ripples' can also be observed in rodent models of schizophrenia (see, e.g., (Buzsáki, 2015) and the references therein), which is the subject of the next subsection.

\subsubsection{Schizophrenia}

Definitions of ASD and schizophrenia have changed over time. In approximately 1950, ASD, which was first described by Kanner (1943) and Asperger (1944), was considered an early version of schizophrenia (Bender, 1947) or a central feature of schizophrenia (Bleuler, 1950). The community started to consider them different disorders in approximately 1970 (Rutter, 1972), since ASD starts during childhood and is characterized by deficits in social interaction and communication, whereas schizophrenia typically has a later onset and is characterized by psychotic symptoms.

However, the early negative symptoms of schizophrenia, such as odd behavior and social withdrawal, communication problems, restricted speech, and poor emotional interactions, are very similar to those of ASD. Furthermore, there are genetic links between ASD and schizophrenia; see, e.g., (Pina-Camacho, Parellada, and Kyriakopoulos, 2016) and the references therein.

Regarding the prominent features of schizophrenia, it appears to affect both men and women at similar rates and has positive and negative symptoms. Positive symptoms include fragmented perception, erroneous binding of features, delusions, hallucinations, and racing thoughts. Negative symptoms, such as apathy, blunted responses, reduced speech, social withdrawal, and others, may also appear.

Different proposals have attempted to connect or separate ASD and schizophrenia. For example, Chisholm et al. (2015) list diverse models that try to explain the frequent co-occurrence of ASD and schizophrenia, including (i) the multiformity model, which suggests a similar underlying disorder but with different expressions; (ii) the increased vulnerability model, which suggests that having one of the impairments increases the likelihood of developing the other; (iii) the model assuming that ASD and schizophrenia are different stages of the same disorder; and (iv) the independence model, which proposes that when the two disorders co-occur, a third distinct nosological condition actually exists (Larson et al., 2017).

Brain waves are also considered the underlying reason for schizophrenia. In this case, the autoencoder model of component formation requires precise synchronization via delay compensation and model-based prediction. When brain wave-driven synchronization is corrupted, learning may be considerably impaired. Thus, imprecisions in brain waves may deteriorate component learning and thus may produce ASD symptoms. We shall examine impairments in the concordance of brain waves and the animal models related to the working of the entorhinal-hippocampal loop and the memory consolidation processes.

As previously mentioned, evolution has found a complex solution for learning and consolidating new memories in mammals. The solution applies a hierarchy of brain waves that has undergone only slight variations during evolution despite the orders of magnitude of increases in brain weight (Buzsáki and Watson, 2012; Buzsáki, Logothetis, and Singer, 2013). Waves include slow waves, theta, alpha, and gamma waves, spindles and ripples. This seems to represent a delicate coordination of machinery that may induce comodulation of the power of faster oscillations in both connected and non-connected areas and regions (Buzsáki and Watson, 2012). In schizophrenia, the meticulous design of brain wave hierarchy and the process of coupling brain waves seem to be impaired in diverse ways (Uhlhaas and Singer, 2006; Uhlhaas et al., 2006; Uhlhaas and Singer, 2012).

There are many constituents involved in manipulating and forming memories. For example, the power of beta and gamma waves increases, and synchronization enhances during the maintenance of working memory, focus of attention processes, perceptual closure, and feature binding, as reviewed by Buzsáki et al. (2013). However, it has been found that induced gamma oscillations are reduced in schizophrenic patients and, to some extent, in their non-schizophrenic siblings, too (Grützner et al., 2013). Some reasons underlying the problems with gamma waves are related to the downregulation of GABA-synthesizing enzymes (Lewis, Hashimoto, and Volk, 2005), NMDA receptor hypofunction (Javitt, 2009), and interactions between NMDA receptors and gamma waves(Hong et al., 2010).

Other oscillations, such as sleep spindles and theta and gamma coupling, are also affected in schizophrenia patients, as shown by (Ferrarelli et al., 2010) and (Lisman and Buzsáki, 2008), respectively. Brain wave imprecisions across large distances may arise from peculiarities and changes in network structure (Cocchi et al., 2017) and/or in the speed of information propagation, which is determined by axon caliber and myelination; see, e.g., (Innocenti, Vercelli, and Caminiti, 2013) and the references therein. We shall address these points later.

We end this subsection with a few notes regarding similarities and dissimilarities in binding and lowcomplexity component formation, respectively, in relation to the self in ASD and schizophrenia.

1. Binding problems have been observed in ASD, e.g., in pairing audio and visual signals and in binding interoceptive signals (Noel et al., 2018). Binding requires precise temporal windows, but experiments with ASD patients show expanded audiovisual temporal binding windows and completely diminished temporal acuity for perceiving cardiovisual (interoceptive to exteroceptive) information 
Colors of Autism Spectrum:

(Noel et al., 2018).

2. Errors in binding give rise to errors in predictive model learning. Self-tickling is an example. The insensitivity to self-tickling should not be surprising given a precise model of the self, and indeed, typical individuals cannot tickle themselves. This remains the case even if bodies are swapped in the body transfer illusion (Van Doorn, Hohwy, and Symmons, 2014). However, the perceptual consequences of self-produced touch are more ticklish in individuals with Asperger's syndrome than in normal subjects (Blakemore et al., 2006), and self-tickling is particularly successful for individuals with pronounced schizotypal traits (Lemaitre, Luyat, and Lafargue, 2016).

3. Experimenting with the fast malleability of the body representation into the environment, as in the rubber hand illusion, Noel et al. (2017) found that patients with schizophrenia and ASD behave very differently. Schizophrenia patients had a weak or variable bodily boundary between the self and the environment, whereas ASD patients had a sharp boundary. We note that the complexity of the separation of the self from the environment corresponds to the separation of self-controlled components from the rest of the world; thus, such separation seems relatively simple among the problems of component learning. In turn, for ASD patients, much less impairment is expected in this low-complexity task compared with higher-complexity tasks. However, since learning is more focused on the self than on partners, learned boundaries may be more rigid for individuals with ASD.

These findings support our proposal that the impairments of ASD manifest in high-complexity cognitive tasks. In particular, binding can be impaired, but separation of low-complexity components seems less sensitive in some cases and it may be spared.

\subsubsection{Attention deficit, hyperactivity disorder}

Attention deficit hyperactivity disorder (ADHD) has the highest incidence rate among all neurodevelopmental disorders; it affects $>10 \%$ of the children in the USA (Duda et al., 2017). ADHD and ASD were mutually exclusive in the Diagnostic and Statistical Manual of Mental Disorders [4th ed.; DSM-4; American Psychiatric Association, 1994]), and simultaneous diagnosis was excluded. This has changed in the DSM-5 due to the high comorbidity ratio. However, they differ; deficits in attention, impulsivity and high activity levels characterize ADHD and are is independent from social context, whereas social communication and interaction are impaired in many ways in ASD.

These are considerable differences between the two disorders, and their comorbidity seems hard to explain. We argue that this is not the case, although the differences are further emphasized in the emotional dimension, which is critical in ASD. ADHD and ASD deviate in terms of narrative production in children; children with ASD performing worse on narrative production, whereas children with ADHD perform better than typically developing (TD) children in terms of their mean Z-scores for emotional and cognitive terms in lexical semantics. In contrast, children with ASD and ADHD are similar in some ways; e.g., in measures of mean length of utterance, they both perform poorly compared with TD children (Kuijper et al., 2017).

An apparent difference is that focus-of-attention is a top-down constraint on bottom-up information flow, and top-down accounts - according to Robertson and Baron-Cohen (2017) - are not 'immediately compatible' with the empirical findings on ASD, since these findings primarily concern changes in low-level, bottom-up sensory processing.

Our model may resolve this apparent dichotomy between the high ASD-ADHD comorbidity and bottom-up (ASD) and top-down (ADHD) characteristics: Component formation involves the autoencoder (Lörincz and Buzsáki, 2000; Lőrincz and Sárkány, 2017) and requires bottom-up and recurrent collateral and, finally, top-down processing. In turn, problems with top-down processing may involve impairments in component formation and, in turn, impairments in bottom-up computations. In any autoencoder model, the success of top-down processing depends on the efficiency of memory consolidation, which is a prerequisite for successful bottom-up processing since top-down encoding is the first step in sparse representations and is followed by sparse representation-supervised fast bottom-up estimations (Sprechmann, Bronstein, and Sapiro, 2015; Lőrincz et al., 2016). In turn, ADHD impairments of top-down processing may contribute to ASD symptoms. New results detailed below support this argument.

According to a recent review (Ramtekkar, 2017), there are considerable similarities between the sleep problems experienced in ADHD and ASD and in the memory consolidation problems in both disorders. The increased REM sleep latency present both in ADHD and ASD (see (Ramtekkar, 2017) and the cited references therein) is one of many similarities, and REM sleep has been considered important for memory consolidation (Siegel, 2005; Diekelmann and Born, 2010). However, its direct role has been debated (Stickgold and Walker, 2005). Recently, the picture has become clearer:

(1) Rasch et al. (2007) provided evidence of the causal role of memory consolidation and hippocampus-driven neocortical reactivation during sleep, but

(2) reactivation was observed mostly during slowwave (SW) sleep and not during REM sleep; see (Born and Wilhelm, 2012) and the cited references therein, and

(3) SW and REM sleep have complementary roles in emotional memory consolidation (Cairney et al., 
Colors of Autism Spectrum:

\section{4), whereas}

(4) REM sleep theta rhythms seem necessary for contextual memory consolidation (Boyce et al., 2016).

In turn, the consolidation of contextual memory formation can be impaired in many ways, including when SW sleep is reduced (which occurs in ADHD but not in ASD (Ramtekkar, 2017)); when REM sleep latency is increased, which occurs in both ADHD and ASD (Ramtekkar, 2017); and when REM sleep theta rhythm is impaired (Boyce et al., 2016).

Contextual memory consolidation is critical for component learning since the context disambiguates the meaning of the component (Fig. 2). In turn, we believe that ADHD can be accompanied by ASD due to top-down memory consolidation problems. This aspect of autism may be well approximated by Happé and Frith's weak central coherence (WCC) hypothesis (Happé and Frith, 2006), although the full story seems slightly more complex, since WCC is concerned with impairments in component manipulation, an ADHD like problem, but component formation is a prerequisite for proper manipulations. In turn, we propose that the consolidation and exploitation of components comprise the link between ADHD and ASD.

\subsubsection{Synesthesia}

Literature on the co-occurrence of autism and synesthesia is limited. Baron-Cohen et al. (2013) report that the incidence of synesthesia, in which a sensation in one modality involves perception in another one, is approximately three times higher in autistic adults than in normal subjects. Hughes et al. (2017) found that synesthesia in autism is linked to savant skills. These results are further supported by Ward et al. (2017), who found that synesthetes have enhanced perception and attention and exhibit autistic-like impairments, too. It has been found that axonal connections between V4, which is involved in color processing, and the so-called 'grapheme area' are denser in synesthetes than in controls (Rouw and Scholte, 2007). Both of these areas are in the fusiform gyrus, and some portions can be adjacent. Other findings support a cross-activation model (Ramachandran and Seckel, 2015) between these areas, and some of these cross-activations seem to be preconscious (Ramachandran and Seckel, 2015). In turn, the comorbidity of autism and synesthesia seems to arise from network effects, possibly as a result of increased axial diffusivity, which can be fostered by enhanced perception and attention to either colors or graphemes.

\subsection{Environmental effects: Discordant monozygotic twins}

Our assumption that component learning and component exploitation are the underlying impairments in autism involve a falsifying issue on environmental effects beyond genetic causes since component learning is inherently connected to experiences and related successes and failures. Due to the numerous constituents of component formation and manipulation, such as the speed of processing, the growth features of the network, the sparseness of the neural activities, and the interplay between the neocortex and connected structures, including the amygdala, hippocampus, the striatum and the cerebellum, one expects considerable environmental variation, especially among newborns with different life conditions.

As detailed by Mandy and Lai (2016), geneenvironment interactions and gene-environment correlations affect ASD risks in many ways as early as the prenatal period; additionally, there are strong postnatal factors, including caregiver-infant interactions and deprivation, that can also increase the risk of ASD.

The findings of Mandy and Lai (2016) are supported by research involving discordant twins, both monozygotic and dizygotic. Their development can be influenced by obstetric factors, first-year infections, and other events (Willfors et al., 2017). Significant effects can be caused by early medical events, and dysregulations, including feeding and sleep abnormalities, crying, and worriedness. Among the many candidates, the strict ratio of the frequencies of brain oscillations, the large size of the human brain, and the need for communication and synchronization among brain regions highlight the environmental effects on (i) myelination (Forbes and Gallo, 2017), (ii) the connected gyrification processes (Kates, Ikuta, and Burnette, 2009; Ecker et al., 2016; Yoshida et al., 2017), (iii) potential increases in brain volume (Hazlett et al., 2017), and (iv) the developmental trajectories of frontal networks (Catani et al., 2016). Such interactions are complex; their effect may depend strongly on other vulnerabilities as emphasized by Beauchaine and Constantino (2017), and they all can influence component learning and manipulation.

\subsection{Implicit learning, control, and proce- dural learning}

According to many models, including (Burgess, Recce, and O'Keefe, 1994; Lőrincz and Buzsáki, 2000; Franzius, Sprekeler, and Wiskott, 2007; Lőrincz and Szirtes, 2009; Lőrincz and Sárkány, 2017; Finnegan, Shaw, and Becker, 2017), among others, one of the tasks of the hippocampus is the discovery of components. Furthermore, and importantly, learned components are to be 'wired into' the neocortical substrate as declarative (explicit) memory. Declarative memory has two forms: Semantic memory and episodic, or autobiographic, memory, which allow the individual to learn and recall experiences in the context of space and time.

If component learning is corrupted, then explicit memory can be impaired in diverse ways, including 
the encoding and retrieval processes. However, under our model assumptions, implicit memory does not have to be involved, at least not directly. It is then a falsifying issue to determine whether implicit memory has any specificity for autistic individuals. The prediction of our model is that implicit memory is greatly independent from explicit memory.

Experimental findings indicate that implicit learning is indeed intact in ASD (Renner, Klinger, and Klinger, 2000; Brown et al., 2010; Németh et al., 2010; Ring, Gaigg, and Bowler, 2015). We note the following:

(1) For a full account, age-related changes should also be considered (Zwart et al., 2017).

(2) There are some conflicting findings in ASD individuals regarding statistical learning, which operates mostly implicitly. Arciuli (2017) suggested that divergent findings be resolved by considering the multi-functional needs of statistical learning. Arciuli's arguments exploit the studies by Brown et al. (2010) that indicate that the degree of communication impairment is independent from performance on implicit learning tasks, in contrast with group-level comparisons with different age groups.

Statistical learning is accompanied by different phenomena, including repetition enhancement/suppression and aftereffects. Repetition enhancement and suppression have been observed after repeated presentations of the stimulus; neural responses change with repetition. Since repetition suppression and repetition enhancement occur within the same region (De Gardelle et al., 2012), it has been hypothesized that repetition promotes predicted activities and suppresses prediction errors, and the former are sparse. This is consistent with the sparse coding principles that we emphasize in our model, in which error suppression and representation enhancement accelerate computation. According to Ewbank et al. (2014), repetition suppression is weaker for individuals with greater autistic impairment. The intriguing finding is that repetition suppression for faces also diminishes if faces are remembered as opposed if they are forgotten (Xue et al., 2011), raising the questions of whether (i) learning is faster or (ii) the effect is weaker and learning is slower, or (iii) both cases may occur in autistic individuals.

The aftereffect is distinguished from repetition suppression; it is characterized as a behavioral response related to classification. For example, face aftereffects occur upon exposure to an adaptor face that causes decisions to move towards the opposite characteristics; e.g., upon adaptation to a wider face, a normal face appears narrower, or if the adaptor face has strong male characteristics, a neutral face will be judged more as a female; see, e.g., (Kovács et al., 2007) and the cited references therein. There are many similar findings, including facial expressions, among others; see, e.g., (Kovács et al., 2007; Hills, 2013; Morgan, Schreiber, and Solomon, 2016) and their cited references. According to Cook et al. (2014), facial aftereffects related to identity and facial expressions are intact in highfunctioning adults with autism. Since the literature presents different results and since the aftereffect is weaker in autistic children than in normal subjects, the authors note that such changes may not be a lifelong feature of the condition (2014) but could be due to slowed learning. We shall return to the issue of slow learning later.

In the alternating serial reaction time task, a procedural learning task, children with grammar learning deficits were compared with typically developing children (Hedenius et al., 2011). Both groups showed evidence of initial learning, but consolidation occurred only in the typically developing children. The grammarimpaired group appeared to lose previously gained knowledge of sequences, and there was a correlation between grammar-related knowledge and procedural learning. We propose the following explanation: Children's grammar-related knowledge can be viewed as a compressed structure comprising many individual examples, and such compressed explicit knowledge may alleviate the consolidation process as opposed to the consolidation of individual examples separately.

\subsection{Slowed learning of components}

The speed of learning in declarative memory, including semantic and episodic memory, is another falsifying issue for us. Our model predicts that component learning is impaired and thus learning is slower and this is more so for more complex tasks. We first describe a specific case related to component formation and then present a more general discussion of episodes and related communication.

The case of Nadia is a very special and revealing example. Nadia was an autistic savant whose horse drawings during early childhood were highly artistic (Selfe, 1977). These drawings were somewhat similar to Leonardo da Vinci's horse drawings, as shown in Figs. 1.4 and 1.5 in (Selfe, 2012). Drawings provide special information; in contrast with Nadia's drawing, a typical child's drawings emphasize the components and present prototypical views of those components (Snyder and Thomas, 2001). Nadia's drawings are more like photographs; in them, it is difficult to tell whether the horse has eyes or how many legs the horse has, and irrelevant and uncompleted details are added. However, by the age of 20, Nadia could draw childish drawings! For an example, see Fig. 2.46 in (Selfe, 2012). It seems that for Nadia, component learning was active, but it was very slow compared with that of typically developing children.

Language is a very special part of cognition. By definition, it serves as a compressed representation of episodes, facts, and rules. Words or even sentences are typically ambiguous, and even when sentences have perfect syntax, they may not make sense if the con- 
text is not known to a member of the conversation; consider Fig.2, for example. The context can come from previous sentences or joint experiences, or the sentence may refer to an actual environmental situation not known to a remote listener. The distributional hypothesis in linguistics - that words that occur in the same context tend to support similar meanings (Harris, 1954) - shows the relevance of context. In addition to its episodic nature, language uses components and creates a combinatorial set of potential expressions. In turn, any component may need other components to specify its meaning. Thus, a complete utterance with the same intonation should have the same consequences in the same context. In turn, one does not need to know the compositional nature of language to use a specific utterance for a specific purpose in a specific context. Such repetitions take advantage of language, but only as signals and not as a compositional combinatorial structure.

An example of the simplification of the combinatorial nature of language is if in a dialog, the speaker refers to her- or himself in third person, especially if the third-person reference is primed by the previous sentence and/or by previous dialog. In such cases, the third person version may come more easily than a firstperson one. The case that somebody refers to himself in the third person is frequent in autism.

\subsection{The self as a component}

Our basic assumption is that social interaction is complex because it includes a considerable number of components, because the partner(s) components are latent, and because the latent components might have strong inter-individual correlations, e.g., feelings of happiness, anger, or fear. Important steps for the proper control of social behavior are (a) the separation of components belonging to different participants and (b) the concurrent and goal-oriented adaptation to or manipulation of those components. The separation of the self as a controllable entity is thus critical since it simplifies the control-related tasks in the interaction: The individual needs to predict the partner(s) and then decide how to act accordingly.

Some findings are support our hypothesis that impairment in component learning and manipulation can influence predictive capabilities and prediction-based actions during social interactions for people with ASD. For example,

- according to recent studies concerning highfunctioning autistic individuals, the ability to predict the actions of others is impaired, but the perception of actions of others is not (Von Der Lühe et al., 2016),

- high-functioning autistic individuals showed no evidence of impairments in the so-called 'sense of agency', which refers to the experience of the self as the 'agent' executing 'its' own actions, but did show deficits in perspective taking (David, Newen, and Vogeley, 2008).

We should add that the sense of agency is less impaired than the proper model of the self. The former can be intact while the latter can be impaired in schizophrenia and in ASD, as discussed previously in the example of self-tickling. In turn, the self itself is a sophisticated component, especially in interactions with partners that have latent variables.

\section{Discussion: Comparisons with other models}

In their review, Robertson and Baron-Cohen (2017) ask whether it is time to abandon the idea of a unified account of autism. We put forth a model in this paper and say, 'No'. Here, we discuss why. The model is supported by recent advances in neuroscience and artificial neural networks. It is based on the following ideas:

- autism is rooted in a collection of impairments,

- the actual collection of impairments may differ for each impaired individual,

- the common feature of these collections is that elements of them are related to the cornerstone of intelligence, the curse of dimensionality (Bellman, 1957) and its consequence, the need for explicit memory in cognitive systems with small memory spans,

- diversity of the elements arises from the constraints of explicit memory formation in the connectome, a distributed neuronal system with relatively large delays, and

- no other psychiatric problem dominates the impairment.

We provide details below and relate our model to others. We start by noting that our sensory spaces have huge dimensions, on the order of million(s), whereas our memory span for cognition is between 5 and 9 items and thus only a few elements (components) of the representations at a time can be used for cognition. The better the representation, the smaller the search problem for cognition, the more it fits within this constraint and the faster the solution is. Behavioral feedback can shape the representation for improved performance and can optimize behavioral patterns, including future objectives and goals.

For any individual, there are two basic ways to proceed for success:

(1) Choose tasks that you know well and optimize action series further; gain more practice. This is the direction of optimizing procedural memory.

(2) Choose tasks that you are interested in but do not know (well) yet. Find solutions, try to simplify them, think flexibly about the subtasks, find the 
Colors of Autism Spectrum:

simplest issue to solve and make these solutions robust so that they can address different situations.

Now, the dilemma is as follows: The tasks that we solve depend on us and on the environment. We prioritize the resources for solving emerging concurrent tasks. The order of the tasks influences the collection of experiences and thus the learning from errors, and, depending on the tasks, we may become more knowledgeable or more precise, and possibly both. Practice requires time, and improvements can be measured. Note, however, that many cognitive problems differ since they are either solved or not and there is no related simple metric, consider chess, for example. This is not the case for mental models about dynamical systems, like the body, or the environment. Searches in the cognitive space can be impossible or very hard depending on the complexity of the problem that can be simplified by novel concepts if those are understood. For example, prediction in the Ptolemaic model is difficult, and a different coordinate system needs to be discovered and Newton's Laws should be mastered to practice predictions with high precision. In this regard, it is an intriguing issue that in rats, there is a special mechanism for the learning of diverse coordinate systems and the related metric (Taube, 2007) and that mechanism requires head direction cells. Indeed, the head direction cells can provide supervisory information for the formation of both place cells and grid cells (Lőrincz and Sárkány, 2017), supporting high level cognitive tasks, like homing.

The two ways, i.e., (1) and (2), work best if they are combined for an efficient description of the task space, to find proper solutions by thinking, to join the elements of the found solutions into controlling 'macros', i.e., longer procedures that can be practiced. Rules and theorems are somewhat similar to macros. They also require searches and practice, but in the cognitive space. Until new components and macros are discovered, one is left to explore new solutions or exploit the old ones. When the latter is the main method for problem solving, behavior seems rigid.

Our proposal for a unifying view of autism is that in autism, both point (1) and point (2) are impaired in numerous and cumulative ways. This corrupted nature becomes more visible in the most complex problems that (i) have many variables and (ii) include hidden variables. Social behavior is such a problem; it requires both the estimation of many components for joint planning and practice for joint task execution. Without the proper components for social interaction, thinking takes a long time, and decisions become risky when compared with established old procedures or routines that work in simpler cases. In turn, the essence of our thinking as we mentioned previously is that autism is the tip of the iceberg; autism is the symptom of comorbidity of many underlying cumulative impairments in component formation and component manipulation capabilities.

There are a number of 'algorithms' required by intact component formation and manipulation. We listed some previously (at the end of Section 2) and now consider them in this discussion. We proceed as follows: We consider different theories of autism and address their strengths and weaknesses from the point of view of experimental findings and considerations regarding component formation and manipulations.

\subsection{ASD models: Strengths and weak- nesses}

There are many models of autism that have both theoretical and experimental supports. Some of them are more involved in information theory and are supported by diverse experimental findings, like the Bayesian Prior Theory. Other models have more heuristic flavor, like Weak Central Coherence Theory. However, another set of models, such as Inflammation Theory, is based on specific, direct experimental findings that try to derive ASD symptoms as consequences. We review and discuss such models in this subsection. We also comment on them from the point of view of our model.

\subsubsection{Noisy brain and excitation/inhibition ratio imbalance}

Learning is harder in noise since either the arbitrary noise is also learned and remembered, which negatively impacts recognition and generalization, or the noise must be filtered, but perfect filtering is a demanding task and part of the relevant information may be eliminated during filtering. Rubenstein and Merzenich (2003) suggested that ASD is the result of noise in the brain. The proposal seems reasonable in a network using sparse representation, such as the brain. For example, noise arises if sparsification is inefficient, which points to the potential role of GABAergic feedforward and feedback inhibitions, as will be discussed later. However, there is both supporting and conflicting evidence, suggesting that the picture is complex.

Markram et al. (2007) studied the valproic acid rat model of autism in their search for a unifying theory of autism. Valproic acid is an anticonvulsant and mood stabilizing drug that has been used for epilepsy and schizophrenia. Markram et al. proposed that hyperfunctionalities in reactivity, plasticity, perception, attention, and memory become debilitating in ASD, causing social and environmental withdrawal and locking the individual into a small repertoire of proven routines. In other words, the brain is not noisy, but it is highly responsive, and strong influences are dampened by restricted behaviors. Based on their studies, they propose that excitations are too high and have behavioral consequences.

Davis and Plaisted-Grant (2015) argue that ASD symptoms reflect too little instead of too much neural noise. They argue that (i) the stochastic resonance 
observed in single unit recordings (see the references they cite) can take advantage of additive noise and may give rise to improved detection and discrimination thresholds; (ii) noise facilitates transitions between observations, but such transitions can be slow or even missing in binocular rivalry for ASD, as reported in (Robertson et al., 2013). Furthermore, (iii) given similar inputs belonging to the same category, generalization between the inputs may become easier if the noise level is higher, a well-known algorithmic method used in nonlinear denoising autoencoders (Vincent et al., 2008); however, generalization processes seem inefficient in autism (Plaisted, 2000; Plaisted, 2001).

Dickinson et al. (2016) reviewed the literature on excitation-inhibition balance in ASD and found that imbalances are critical and there is supporting evidence for such changes, but taken together, the evidence justifies neither a net increase in excitation nor a net increase in inhibition in autism. David et al. (2016) consider not the strengths but the variability of cortical oscillation patterns. They note that searches for abnormal power spectra provide inconsistent results in autism. They emphasize that trial-to-trial variability in cortical oscillations form operational noise in neuronal networks that should consistently communicate between remote areas, and such variations have been found in ASD.

Recent studies of infants with ASD led to intriguing novel findings on cascades of network efficiencies (Lewis et al., 2017). Network inefficiencies were found in infants at high risk of later ASD before symptom consolidation. Inefficiencies - detected by MRI seedbased tractography measures of connection length and strength - were first apparent in low-level sensory processing as early as the age of 6 months, but only in short-range cortico-cortical connectivity. Inefficiency then spread to higher-level processing, and ASD symptoms appeared. Symptom severity can be predicted by the inefficiencies measured much earlier in low-level processing. Lewis et al. suggest that children with ASD may suffer from diminished synaptic pruning during early development. In his comments, Winterer (2017) aimed to connect network inefficiencies to the endogenous neuronal noisy brain found in schizophrenia; see, e.g., (Winterer et al., 2004), and (Peterson et al., 2017) as well as the references cited. In sum, endogenous noise and excitation-inhibition imbalance in the context of ASD are controversial but seem to be present in different cohorts measured in different ways and with different extents of ASD symptoms (Dickinson, Jones, and Milne, 2016).

Regarding component formation and the cognitive manipulation of components, both noise and hyperfunctionality can seriously impair them in many ways since component formation presupposes generalization abilities and the development of the precise metric for the components, i.e., discrimination capabilities, whereas manipulation requires precisely crafted, wellsynchronized means of control.

\subsubsection{Bayesian Prior Theory}

Pellicano and Burr (2012) suggested the use of Bayesian models to understand autistic information processing. According to Pellicano and Burr, differences lie in the perceptual mechanisms; namely, people with ASD have 'hypo-priors' that give rise to unique, highly precise perceptual experiences. Based on this assumption, Pellicano and Burr explain many autistic characteristics, from sensory processing to non-social impairments.

A recent work by Palmer et al. (2017) summarizes the proposal that in autism, sensory information has larger weighting than in normal people. Palmer et al. argue that balance between perception and action may be the characteristic difference between people with autism and normal people in both social and non-social behaviors. Since action and related learning are connected to procedural memory and thus to repetition priming, repetition suppression and component learning and manipulation, the complexity of impairments and the escalation of problems during development are beyond our consideration.

There is little doubt that Bayesian inference is difficult to discount, and one may ask whether this is the strategy applied by the brain. At first glance, the answer seems to be yes; see, e.g., (Berkes et al., 2011; Lee and Mumford, 2003; Robertson and Baron-Cohen, 2017) and the cited references therein.

One may wonder what the winning learning and working strategy should be in a near-deterministic world when deterministic actions with deterministic outcomes are possible and are desired. Experimental findings on endogenous and exogenously modulated binocular rivalry (Brascamp et al., 2018) seem to contradict simple Bayesian principles, since a Bayesian observer would always pick the higher-probability interpretation. On the other hand, the fact that binocular rivalry is slowed down in autism seems consistent with the Bayesian observer assumption. The switching phenomenon, however, indicates that simple Bayesian models are incomplete.

\subsubsection{Mirror Neuron Theory}

Since the discovery of mirror neurons (Gallese et al., 1996; Rizzolatti et al., 1996), that react similarly for goal-oriented self-motions and for similar motions of others and thus allow estimations of the intentions of others, scientists have considered that the mirror neuron system may be impaired or possibly dysfunctional in autism (Williams et al., 2001). In other words, it was thought that in typical cases, mirror neurons can provide supervisory information for training and for copying behavioral templates, as in the case of successful models of the learning of dynamic movement primitives (Schaal, 2006; Ijspeert et al., 2013; Chen et al., 2015).

Experiments support this assumption to some extent: 
$\mu$ waves are typically blocked or reduced during voluntary muscle movement, e.g., when opening or closing the hands, regardless of whether the subject makes the movement or observes someone else making, but they were not blocked even in high-functioning autistic children when they monitored someone else's muscle movement (Oberman et al., 2005).

These findings on $\mu$ waves used to be considered compelling (but, in our view, only implicit) evidence of problems with the mirror neuron system (Ramachandran and Oberman, 2006). However, doubts have remained, and contradicting evidence has been found. For example, (Bird et al., 2007; Sowden et al., 2016) showed that automatic imitation is intact in ASD. Furthermore, upon separating automatic imitation from spatial compatibility effects (i.e., separating responses on the same side and on the opposite side), there was no relationship between spatial compatibility and autism symptom severity, meaning that individuals with ASD exhibited increased (and not decreased) imitations. The phenomenon is called hyperimitation (Bird et al., 2007; Spengler, Bird, and Brass, 2010; Sowden et al., 2016; Deschrijver, Wiersema, and Brass, 2017). These findings are supported by evidence that individuals with ASD frequently engage in strong imitative behavior, such as echolalia and echopraxia.

We note that component learning should support the development of hand representations independent from the owner of the hand to decrease the curse of dimensionality. However, imitation does not require this separation. It is possible that the representation of the hand works well, so that representation is separated from the representations of other body parts, but the representation does not have to 'know' whether that hand belongs to the self. For example, thinking in terms of the where and what pathways, the what system may know that it is hand, the where system may know where that hand is; however, these or other subsystems may not know well whether that region of the peripersonal space as defined by Graziano (2006) belongs to the self or not, or they may know it well, but the fusion of that information is corrupted with respect to the hand. Lacking this piece of information, imitation becomes similar to repetition, and that might account for the $\mu$ wave-related findings.

\subsubsection{Weak Central Coherence Theory}

Weak Central Coherence (WCC) theory has been an insightful model for autism. Happé and Frith (1994) put forth the idea that autistic behavior is the result of impairments in extracting global form and meaning. Later, the model was modified (Happé and Frith, 2006) to say that problems might arise from the superiority of local processing, which is a bias in the processing strategy, and weak coherence may not be the cause but a symptom of autistic behavior. Robertson and BaronCohen (2017) object to the theory on the grounds that it is a top-down mechanism and that bias in the cognitive strategy can hardly explain low-level sensory processing problems.

We note, however, that component formation and manipulation can exploit either holistic recognition (Van Belle et al., 2010), or 'recognition-by-components' (Biederman, 1987), or both. The applied strategy should be context dependent, and as such, it is restricted if the quality of formed components is poor. In turn, the presence or absence of components or the quality of the learned components does influence cognitive strategy via the feedforward estimation of the available sparse components (Sprechmann, Bronstein, and Sapiro, 2015) and (Lőrincz et al., 2016). In addition, if holistic, i.e., more complex, recognition is impaired, then component-based recognition followed by inferences may become typical that helps to train the holistic learning process as illustrated in (Lörincz et al., 2018).

\subsubsection{Going deeper: Specific models}

\subsubsection{Delayed development in multisensory pro- cessing}

Our proposal that component formation and manipulation are corrupted in ASD means that learning is immature, but components may improve with time. In other words, if ASD is an impairment of the neural learning machinery, then special, topic related impairments may diminish with time. For example, if anxiety and fear do not interfere or prevent social interactions, then social behavior should improve with time. This is different from other types of impairments, such as color blindness that may not change. Developmental trajectories can provide support for such thinking.

A recent paper (Beker, Foxe, and Molholm, 2018) details this direction and suggests remediations. Beker et al. put forth arguments about multisensory processing. They reviewed studies on sensory integration deficits at different ages that diminished or sometimes disappeared over time. Decreases in such sensory integration deficits have been found for lower-level and higher-level speech processing, such as the McGurk effect, which can be observed for short plosives; see the discovery (McGurk and MacDonald, 1976) and a review (Alsius, Paré, and Munhall, 2017) of the effect and the related findings on the dependencies of the autism quotient (Frith, 1991; Woynaroski et al., 2013; Bebko, Schroeder, and Weiss, 2014; Ujiie et al., 2015),

Beker et al. (2018) examined lower- and higherlevel sensory integration phenomena, from flashes and beeps to speech and gesture, and found that most of them support slower development. Delayed multisensory processing can explain several symptoms found in ASD, but sensory integration is only a part of component formation. Some aspects of autism are not addressed if one assumes that this type of impairment is the only underlying impairment of ASD. Impairments in multisensory processing can be due to impaired 
recognition capabilities in any modality or to impairments in delay compensation in the different processing channels. None of these features alone can explain why social learning and language are impaired the most. However, impairments in component formation and exploitation predict the slowed learning of multisensory information fusion, and any slowing down in learning supports our model.

\subsubsection{Role of inflammation}

Inflammation has been cited in a recent paper (Mazarati, Lewis, and Pittman, 2017) as a cause of epilepsy, ASD, and depression. According to Mazarati et al. (2017), (i) inflammatory markers are elevated in these disorders, (ii) these markers may interfere with serotoninergic pathways, (iii) weakening of the inflammatory pathways can reduce the symptoms of these disorders, and (iv) early provocation of inflammatory pathways produces a more excitable brain with an ASDlike phenotype in animal models. Thus, inflammation can impair and slow down component learning and may induce other impairments. However, inflammation alone cannot explain why the primary symptom of ASD is impaired social relations. On the other hand, inflammation that may intensify autistic features supports our assumption that autism is the result of the comorbidity of different impairments that affect component formation and manipulation.

\subsubsection{The minicolumn hypothesis}

An intriguing and challenging observation has been made by Casanova et al. (2002; 2004; 2006), (Casanova, 2007). They found changes in the minicolumnar structure of neocortical superficial layers. In particular, they found that minicolumns are smaller in the autistic brain.

Minicolumns are the smallest functional units of the neocortex, according to Mountcastle (see, e.g., (Mountcastle, 1997)), and they differ significantly between areas and among mammalian species (Yáñez et al., 2005). Their development also involves double-bouquet cells (DBC), the horsetail-like GABAergic neurons of the superficial layers that underwent a shift during mammalian evolution. It seems that these neurons play an important role in minicolumn organization, but mainly in primates (Yáñez et al., 2005). Furthermore, minicolumns seem to increase with increasing brain mass among anthropoid primates. One may wonder about brain size and minicolumnar variations in ASD. It should also be noted that minicolumns may not have a function at all, according to some proposals in the literature (Horton and Adams, 2005). However, recent studies emphasize the need for further comparative analyses of the structure of minicolumns and their ecological, behavioral, and cognitive correlates (Spocter et al., 2015).
Experimental findings concerning minicolumns are controversial in ASD. While Casanova et al. report decreases in minicolumnar organization in ASD, others have also found differences but reported wider and not narrower minicolumns relative to controls (McKavanagh, Buckley, and Chance, 2015).

Component formation may offer explanations for both types of findings based on theoretical grounds. Minicolumns and their DBCs' tendency to inhibit the pyramidal cells of neighboring minicolumns create an ideal substrate for structured sparse representations, a highly efficient machine-learning approach (Bach, 2010) discussed previously. Here, structure means a group of top-down memories of a generative, i.e., autoencoding, nature. Unique solutions are warranted by sparsification. Slow operation is a drawback, but fast feedforward estimation of the relevant groups can speed up operations (Lörincz et al., 2016). If the number of groups required for proper autoencoding is larger than the dimension of the input (if minicolumns are small), then the operation slows down. Depending on other areas, slower operation may corrupt synchronization between the areas. In the opposite case, i.e., when minicolumns are large, then feedforward selection can be fast, but overgeneralization may occur, and discrimination capabilities and category formation can be impaired. In addition, the smaller number of DBCs may lead to overexcited representations. Such processes could severely impair component learning and component manipulation. These are testable cases.

\subsubsection{Cortical hyperexpansion, brain volume overgrowth, and spatio-temporal con- straints}

Some developmental findings seem relatively general in autism. One example is early brain development (Piven, Elison, and Zylka, 2017). Piven et al. studied high-risk siblings later diagnosed with autism early in their first year and performed neuroimaging studies beyond their first year. The researchers found cortical area hyperexpansion in the first year. In the second year, brain volume overgrowth followed and seemed to be associated with the emergence of social deficits. The researchers reported that hyperexpansion of the cortical surface area occurring during the pre-symptomatic period may co-occur with sensorimotor and attentional deficits (see, (Piven, Elison, and Zylka, 2017) and the cited references therein). The changes, according to Piven et al., alter experience-dependent neuronal development, and give rise to increased neuronal activities and, eventually, brain volume overgrowth. The final result is that the refinement of neural circuitry is disrupted, and autistic social deficits become apparent during the second year of life.

The relevance of these findings is shown in another paper by Piven and colleagues (Hazlett et al., 2017) that was mentioned earlier: within high-risk siblings, information about brain surface area can predict a pos- 
itive diagnosis of autism with predictive and sensitivity values of $81 \%$ and $88 \%$, respectively. This appears to be an important trajectory of autism development in high-risk individuals. Other developmental trajectories within this group and in other groups should be identified for a better understanding of this disorder.

Duan et al. (2017) found specific developmental characteristics in the longitudinal MR images of healthy infants taken at around birth, 1 and 2 years of age. Changes differ at the level of the primary folds, which flatten out, whereas most of the minor folds become more convoluted.

In terms of component learning and manipulation, constraints on 'wiring length' are important since there is no high-functioning connectome without proper wiring. Severe constraints may emerge if the surface area grows slightly faster, making flattening harder and thus (i) restricting the room for minor folds, (ii) increasing the length needed for otherwise short-range connections in the white matter and (iii) eventually increasing the volume of the brain but still restricting the fast communication permitted by the constraints of scale-free small world structures (see, e.g., (Buzsáki and Moser, 2013; Kaiser, 2017; Levina and Priesemann, 2017), and the cited references on scale-free small world features in the healthy brain). In other words, we suspect that gyrification supports the identified scale-free small world wiring, and conflict may arise between learning and normal brain size if it does not.

\subsubsection{Malfunctioning interneurons}

Parallel with the putative excitation inhibition imbalance in ASD, parvalbumin-expressing and other inhibitory interneurons have become of interest. Researchers have found a few subtleties, e.g., in the knockout mouse model of autism:

1. Circuit dysfunction, impaired sensory gating and social disability emerge in conditional knockout mice in which cox10 expression in parvalbumin cells has been eliminated, giving rise to mitochondrial dysfunction in these cells without any loss in the number of cells. The parvalbumin cells could not maintain their firing rates under these conditions, and imbalances in the exhibitory-inhibitory ratio occurred. The resulting behavioral alterations resembled those observed in schizophrenia and autism.

2. Disrupted developmental N-methyl-D-aspartatereceptor (NMDAR) function was modeled in knockout mice (Gandal et al., 2012), resulting in changes in the excitation-inhibition balance due to increased pyramidal cell excitability and selective disruption of parvalbumin-expressing interneurons. Changes in the signal-to-noise ratio in the gamma band were observed, among other disruptions, and deficits in social preference also appeared. In addition to intellectual disability, reduced NMDAR signaling is associated with both schizophrenia and autism.

3. Inhibitory neurons regulate learning in neuronal networks. For example, feedforward and feedback inhibition is influenced by somatostatinexpressing interneurons, giving rise to behavioral state- dependent inhibition and plasticity. In turn, these interneurons integrate different sensory information flows. Parvalbumin-expressing fast-spiking basket cells change their synaptic strengths in synapses targeting the soma of pyramidal cells. According to research findings, fastspiking parvalbumin-expressing interneurons participate in the maintenance of the excitatoryinhibitory balance. Errors in regulation are due to molecular mechanisms and can be counteracted via different means, as reviewed by Dehorter et al. (2017).

4. NMDAR dysfunction is related to ASD, and ASD symptoms can be influenced by modifying NMDAR function in humans; see (Lee, Choi, and Kim, 2015). Furthermore, animal models of ASD indicate that correcting NMDAR dysfunction may have a therapeutic effect on ASD.

5. The number of parvalbumin-expressing interneurons in ASD is smaller than normal in the medial temporal cortex (Hashemi et al., 2017).

6. A low Cl- ion concentration is critical for proper GABAergic inhibitory actions. High concentrations of these ions can give rise to excitatory GABA actions and are characteristic of autism and various types of epilepsy, suggesting another ASD-related comorbidity family that may benefit from special treatments, as suggested by Ben-Ari et al.; see, e.g., (Ben-Ari, 2017; Lemonnier et al., 2017) and the cited references.

7. The GABAergic effect changes from excitatory to inhibitory near birth. This shift seems to be corrupted in autism, and GABAergic dysfunction might be related to cortical area hyperexpansion (Hazlett et al., 2017). Regulation of the shift via oxytocin treatment has been suggested by Ben-Ari (2015).

The molecular interactions that are responsible for different dysfunctions give rise to numerous impairments. However, these dysfunctions cannot explain why and how ASD - that is, an impairment in social behavior - becomes the leading symptom in some cases. Other general problems, e.g., network subtleties to be reviewed later, pose similar questions.

\subsubsection{Cognitive dysfunction}

Cantio et al. (2016) studied cognitive-level symptoms and searched for a universal pattern of cognitive impairments in ASD. They found that two such impairments - (i) impaired theory of mind, i.e., theorizing about 
the hidden mental states of other people, and (ii) impaired executive function, manifested as repetitive and stereotyped behaviors, among other characteristics predict autism at a rate of up to $75 \%$ (50\% being a random association), which is not a very high value. Nonetheless, their results support the idea that the social behavior impairments in autistic individuals may arise from small but potentially different subsets of many potential causes. In the case of impairments in generalization capabilities, e.g., impairments in prototype formation, modification of the input distribution can help, as we review below. Prototype formation is a kind of generalization, and it is probably needed for component learning.

The random dot test is a peculiar example. It was developed by Posner et al. (1967) and showed specific and different performances for hippocampal subjects (Knowlton and Squire, 1993; Squire and Knowlton, 1995) and for Alzheimer's patients (Kéri et al., 2001; Kéri et al., 2002; Heindel et al., 2013). In these studies, highly distorted versions of the prototype are first presented. Then, the subject is informed that examples belong to the same group, and s/he is asked to indicate whether a new input belongs to the same group. The performance of hippocampal patients is intact, but they cannot recognize whether an item has been shown before. In patients with Alzheimer's disease, the probability of low-distortion samples being classified as 'belongs to the group' overcomes that of the prototype. In the case of autism, Froelich et al. (2012) found that prototype formation is intact in ASD, but the classification of the highly distorted samples depends on item ordering, whereas for normal subjects, classification is not affected by item ordering. Church et al. (2015) added that children with ASD who show atypical generalization benefit from learning from a single prototypical member of a category, but not from learning from many examples. These findings suggest that (i) individual examples can be preserved in some ASD cohorts, but these examples may not undergo proper generalization, and (ii) changes in the input distributions can help with learning.

Ring et al. (2017) studied a critical piece of information for us: Whether the hippocampus is involved in ASD. Our proposal predicts that ASD is sensitive to hippocampal impairments. Ring et al. used structural learning, biconditional-discrimination and transverse patterning tasks to examine potential hippocampus-related deficits in ASD. Structural learning requires the learning of spatial, temporal or spatiotemporal relations, i.e., particular combinations of elements/components during experiences, and consequently, this type of learning critically depends on the hippocampus; see the references in (Ring et al., 2017). They found that structural learning was impaired in ASD, but other forms of configural learning were not. Since structural learning is known to be hippocampus dependent, the authors claim that their findings confirm hippocampal involvement in ASD.
Component learning enables the 'recognition-bycomponents' process (Biederman, 1987), which is less fragile (but slower) than holistic recognition; see, e.g., the examples in (Lörincz et al., 2018). An impaired component system forms a bottleneck for the flexible responses required in diverse situations in two ways: (i) practiced solutions are rigid but safe, whereas (ii) novel combinations are hard to develop due to imprecise component formation.

We reviewed cognitive impairments that can be caused by hippocampal dysfunction. The existence of such impairments is critical to our proposal regarding component formation in the understanding of autism since the learning of declarative memory is not possible in the absence of the hippocampus. In addition to learning, long-term storage is also a necessity, and the connectome can have other features that may increase the severity of ASD symptoms.

\subsubsection{Problems in the connectome}

ASD seems to have denser connectivities locally and hypoconnectivity globally (Hughes, 2007). Deviations from optimal network structures may give rise to network inefficiencies and may corrupt information processing in the brain; see, e.g., (Lewis et al., 2017) and the cited references. Lewis et al. emphasize the vicious circle issue in their study. They studied infants between the ages of 6 and 12 months with either high or low risks of having autism. They used diffusion measurements and combined them with longitudinal studies. They found that the high-risk infants had inefficiencies in regions that process low-level sensory information. Since sensory processing of the visual stream takes a long time (Kovács et al., 1999; Kovács, 2000), one would expect that early discrepancies could have longlasting effects on learning and that these effects can accumulate. Problems with low-level processing can arise from bandwidth problems between groups of neurons, making information transfer inefficient; this can give rise to synchronization problems and impair multimodal information processing and integration, among other functions.

These vulnerabilities can be hampered further by local neural structural problems, including the maturation of white matter myelination and axial and radial axonal diffusivities (Deoni et al., 2016; Ecker et al., 2016). Such problems can slow down learning and the consolidation of components. Learning and consolidation impairments may manifest in diverse ways, which we discuss below.

\subsection{Potential side effects of impair- ments of component formation}

Since autism - in our view - is a combination of many impairments that influence component formation and exploitation, one expects epiphenomena to arise from 
the individual (small) corruptions. We provide a few selected examples below:

1. Components of the body should be properly represented to serve the flexibility of motion control and the related synchronization needs. For example, neuronal responses in the macaque motor cortex have some similarities to the place cells of the hippocampus; they have the flavor of components, as shown by Aflalo and Graziano (2007), but for actions. Neurons in the motor cortex discretize the final position of the hand in the Cartesian space around the body independent of the starting position. Such neurons are like the place cells of the 'cognitive map', but they are task related; they discretize the targeted end positions of the limb in peripersonal space and are invariant to the initial position. By contrast, hippocampal place fields discretize the actual position and are invariant to the direction of the body. Impairments in component formation can lead to impairments in the invariant properties and in discretization in general and may lead to imprecise control; that is, to dyspraxia. According to a recent report, ASD involves considerably larger decrement when executing simultaneous as opposed to serial gestures than decrement found in control subjects (McAuliffe et al., 2017). Furthermore, praxis errors strongly correlate with autism severity and not IQ (Kaur, Srinivasan, and Bhat, 2018).

2. There are considerable changes in the basal ganglia, which is responsible for motor skill acquisition, including eye movement, eye-hand coordination, and movement coordination. These ganglia form inhibitory loops that, upon disinhibition, launch motor actions; they also play a role in action inhibition and sequence learning. The complex inhibitory structure of the basal ganglia may be altered in autism since inhibitory neurons are often impaired in ASD. Indeed, volumetric changes and alterations in cell densities in the basal ganglia nuclei have been found in autism; see, e.g., (Subramanian et al., 2017) and the references therein. In autism, the basal ganglia operate differently; the sizes of the substructures, e.g., the putamen, caudate nucleus, globus pallidus can change, and the information flow between these units and to and from the neocortex also changes. According to suggestions in the literature reviewed by Subramanian et al., (2017), repetitive behavior is related to these structures. We believe that a restricted motor repertoire and stereotypic behaviors may also originate from impaired component formation.

3. Repetitive behavior may have some inborn circuitry. Consider babbling, for example. It seems that the initial babbling mechanism is (i) language independent and (ii) independent from hearing or deafness (Locke, 1995). Near the end of the babbling phase, normal infants start to engage in echolalia; they start to repeat phonemes and words learned from others. Echopraxia is another example of an inborn source of repetitive behavior. It is common for normal infants, who produce learned gestures and words spontaneously. For more details on the development of normal infants, see (Pulvermüller, 2018) and the cited references therein. This type of practicing is an efficient learning method known as 'learning by doing' in many disciplines, from psychology to machine learning to education. In the control field, the executed (potentially random) motion is considered the desired motion and becomes a self-generated sample to learn from. Since learning is slowed down in autism, repetitive behavior helps with learning and is rewarding for longer times. Indeed, autistic children also repeat speech and gestures, and the duration of this period of repetition can be greatly prolonged. Nonetheless, different mechanisms may be responsible for repetitive behavior. It is known, for example, that autism-associated neuroligin-3 mutations commonly impair striatal circuits and boost repetitive behaviors (Rothwell et al., 2014).

4. Language is component based and combinatorial, with considerable need for context-based pattern completion and disambiguation; thus, it can be and is corrupted in many ways in autism.

5. The networks involved in reinforcement learning are related to several neurocircuitries, such as the prefrontal-subcortical circuits, amygdala, brainstem, and cerebellum (Schuetze et al., 2017). Such complex circuitries can be affected, and such considerations are beyond the scope of this work. We note, however, that reinforcement learning undergoes a combinatorial explosion in the number of variables, and there is a need for components that describe the actual situation in the context of the actual task. Theory calls this 'factored reinforcement learning', and factors are engineered in the applications (Kearns and Koller, 1999; Szita and Lörincz, 2009). The learning of many factors (here, components), of which a few are sufficient for a given situation (that is, the learning of sparse factor representation) is a not-yet-solved theoretical problem in machine learning.

6. The amygdala, a small subcortical structure involved in the regulation of emotions, has been long suspected to play a role in (some aspects of) autistic behavior. Baron-Cohen et al. (2000) developed the amygdala theory of autism based on findings that the amygdala showed limited activity during mentalistic inferences from the eyes in subjects with ASD compared with healthy volunteers. The volume of the amygdala is larger in children with ASD than in typically developing children (Nordahl et al., 2012). There are additional changes in the amygdala (Weir et al., 
Colors of Autism Spectrum:

2018), some of which are common in autism and in schizophrenia and may give rise to excitationinhibition ratio imbalance in this structure (Gao and Penzes, 2015). Since the amygdala plays a role in reinforcement learning (Schuetze et al., 2017), its overall effect in ASD could be large. Although amygdala dysfunction can explain a broad variety of behavioral impairments, it alone cannot explain the richness of phenomena that occur in ASD.

7. The entorhinal-hippocampal loop has been suggested to be responsible for novelty detection (Lőrincz and Buzsáki, 2000) as an extension of the comparator model of the hippocampus suggested by Vinogradova (1970). Learning, according to these models, proceeds by encoding the observed novelty if it is behaviorally relevant. The aberrant precision account of ASD in the predictive coding model suggests enhanced sensitivity to novelty (Lawson, Rees, and Friston, 2014). On the other hand, any novelty may predict behavioral failure if situations are complex, as is the case for social interactions. Such experiences may give rise to novelty-related fear and, due to reinforcement learning, to the avoidance of such situations, leading to a lack of practice and to autistic behavior.

\section{Conclusions}

In a recent paper, Beauchaine and Constantino (2017) discuss the vulnerabilities with respect to ADHD, autism, and depression, among other disorders. Their position goes beyond the 'classical' endophenotype concept. They claim and argue that neural functions are preferable for the description of these behaviors. We present a single main conclusion for this paper, as follows: autism is the result of diverse vulnerabilities related to component formation and exploitation.

We examined the theoretical underpinnings and considered the behavioral issues and comorbidities that frequently occur in autism. Theoretical considerations and experimental findings support our view. This view harmonizes with that of Beauchaine and Constantino (2017) and Krishnan et al. (2016) since there are many ways in which intelligence can be corrupted within the sophisticated machinery of the mammalian brain. The key observation is that cognition should aim to minimize the variables (components) involved in solving diverse tasks due to the limited memory span and the limited time for searching for a solution in cognitive space.

Component formation can be corrupted in countless ways, all of which have their own names if they happen to be a leading cause of corruption. However, combinations of relatively small impairments can lead to impairment that manifests in higher-complexity tasks. Due to the presence of combinations, diverse comorbidities are expected.
Is social behavior complex? We answer this question as follows: There are few problems that require the exploitation of different, well-formed components at the same time. The harder of these problems require the estimation of hidden and uncertain components. Estimation of mood, capabilities and intentions of other people belong to this group of problems. In turn, diverse impairments of component formation will be more striking in such tasks, and in turn, social behavior can be seriously affected.

The route of vulnerability combined with the idea of component formation has promise for explaining autism. It also presents a challenge for precision medicine. The collection of large amounts of data together with data mining (see, e.g., Loth et al. (2016)) may be a viable solution for identifying the actual causes and intervening according the experienced developmental trajectories, more so if these data can be combined with genetic information (Szatmari et al., 2007). Such data collection efforts have already started; see, e.g., (Autism Spectrum Disorders Working Group of The Psychiatric Genomics Consortium et al., 2017) and the associated references.

\section{Acknowledgments}

The author is most grateful for enlightening discussions to Professor Latha V Soorya on autistic behavioral problems and to Ábel Fóthi on gene related issues. The project has been supported by the European Union, co-financed by the European Social Fund (EFOP-3.6.3VEKOP-16-2017-00001).

\section{References}

Aflalo, Tyson N and Michael SA Graziano (2007). "Relationship between unconstrained arm movements and single-neuron firing in the macaque motor cortex". In: Journal of Neuroscience 27.11, pp. 27602780 .

Alsius, Agnès, Martin Paré, and Kevin G Munhall (2017). "Forty years after Hearing lips and seeing voices: The McGurk effect revisited". In: Multisensory Research 31.2, pp. 111-144.

Amiet, Claire et al. (2008). "Epilepsy in autism is associated with intellectual disability and gender: Evidence from a meta-analysis". In: Biological Psychiatry 64.7, pp. 577-582.

Arciuli, Joanne (2017). "The multi-component nature of statistical learning". In: Philosophical Transactions of the Royal Society of London B 372.1711, p. 20160058.

Arora, Sanjeev et al. (2014). "Provable bounds for learning some deep representations". In: International Conference on Machine Learning, pp. 584-592.

Asperger, Hans (1944). “Die „Autistischen Psychopathen" im Kindesalter". In: Archiv für Psychiatrie und Nervenkrankheiten 117.1, pp. 76-136. 
Autism Spectrum Disorders Working Group of The Psychiatric Genomics Consortium et al. (2017). "Metaanalysis of GWAS of over 16,000 individuals with autism spectrum disorder highlights a novel locus at 10q24.32 and a significant overlap with schizophrenia". In: Molecular Autism 8, pp. 1-17.

Bach, Francis R (2010). "Structured sparsity-inducing norms through submodular functions". In: Advances in Neural Information Processing Systems, pp. 118126.

Balardin, Joana Bisol et al. (2015). "Decreased centrality of cortical volume covariance networks in autism spectrum disorders". In: Journal of Psychiatric Research 69, pp. 142-149.

Baron-Cohen, Simon et al. (2000). "The amygdala theory of autism". In: Neuroscience \& Biobehavioral Reviews 24.3, pp. 355-364.

Baron-Cohen, Simon et al. (2013). "Is synaesthesia more common in autism?" In: Molecular Autism 4.1, p. 40.

Beauchaine, Theodore $\mathrm{P}$ and John N Constantino (2017). "Redefining the endophenotype concept to accommodate transdiagnostic vulnerabilities and etiological complexity". In: Biomarkers in Medicine 11.9, pp. 769-780.

Bebko, James M, Jessica H Schroeder, and Jonathan A Weiss (2014). "The McGurk effect in children with autism and Asperger syndrome". In: Autism Research 7.1, pp. 50-59.

Beker, Shlomit, John J Foxe, and Sophie Molholm (2018). "Ripe for solution: Delayed development of multisensory processing in autism and its remediation". In: Neuroscience \& Biobehavioral Reviews 84, pp. 182-192.

Bellman, Richard (1957). Dynamic Programming. Princeton University Press.

Ben-Ari, Yehezkel (2015). "Is birth a critical period in the pathogenesis of autism spectrum disorders?" In: Nature Reviews Neuroscience 16.8, p. 498.

- (2017). "NKCC1 Chloride Importer Antagonists Attenuate Many Neurological and Psychiatric Disorders". In: Trends in Neurosciences 40.9, pp. 536-554.

Bender, Lauretta (1947). "Childhood schizophrenia: Clinical study of one hundred schizophrenic children." In: American Journal of Orthopsychiatry 17.1, pp. 40-56.

Berkes, Pietro et al. (2011). "Spontaneous cortical activity reveals hallmarks of an optimal internal model of the environment". In: Science 331.6013, pp. 8387.

Biederman, Irving (1987). "Recognition - by - components: A theory of human image understanding." In: Psychological Review 94.2, pp. 115-147.

Bird, Geoffrey et al. (2007). "Intact automatic imitation of human and robot actions in autism spectrum disorders". In: Proceedings of the Royal Society of London B: Biological Sciences 274.1628, pp. 3027-3031.
Blakemore, Sarah-Jayne et al. (2006). "Tactile sensitivity in Asperger syndrome". In: Brain and Cognition 61.1, pp. 5-13.

Bleuler, Eugen (1950). "Dementia praecox or the group of schizophrenias (Translated from German)". In: Oxford, England: International Universities Press.

Born, Jan and Ines Wilhelm (2012). "System consolidation of memory during sleep". In: Psychological Research 76.2, pp. 192-203.

Boyce, Richard et al. (2016). "Causal evidence for the role of REM sleep theta rhythm in contextual memory consolidation". In: Science 352.6287 , pp. 812816.

Bragin, Anatol et al. (1999). "High-frequency oscillations in human brain". In: Hippocampus 9.2, pp. 137142.

Brascamp, Jan et al. (2018). "Multistable Perception and the Role of the Frontoparietal Cortex in Perceptual Inference". In: Annual Review of Psychology 69.1, pp. 77-103.

Brascamp, Jan W et al. (2005). "Distributions of alternation rates in various forms of bistable perception". In: Journal of Vision 5.4, pp. 287-298.

Brown, Jamie et al. (2010). "Intact implicit learning in autism spectrum conditions". In: Quarterly Journal of Experimental Psychology 63.9, pp. 1789-1812.

Burgess, Neil, Michael Recce, and John O'Keefe (1994). "A model of hippocampal function". In: Neural Networks 7.6-7, pp. 1065-1081.

Buzsáki, György (1989). "Two-stage model of memory trace formation: A role for "noisy" brain states". In: Neuroscience 31.3, pp. 551-570.

- (2015). "Hippocampal sharp wave-ripple: A cognitive biomarker for episodic memory and planning". In: Hippocampus 25.10, pp. 1073-1188.

Buzsáki, György and Andreas Draguhn (2004). "Neuronal oscillations in cortical networks". In: Science 304.5679, pp. 1926-1929.

Buzsáki, György, Nikos Logothetis, and Wolf Singer (2013). "Scaling brain size, keeping timing: Evolutionary preservation of brain rhythms". In: Neuron 80.3, pp. 751-764.

Buzsáki, György and Edvard I Moser (2013). "Memory, navigation and theta rhythm in the hippocampalentorhinal system". In: Nature Neuroscience 16.2, p. 130 .

Buzsáki, György and Brendon O Watson (2012). "Brain rhythms and neural syntax: Implications for efficient coding of cognitive content and neuropsychiatric disease." In: Dialogues in Clinical Neuroscience 14.4, p. 345 .

Cairney, Scott A et al. (2014). "Complementary roles of slow-wave sleep and rapid eye movement sleep in emotional memory consolidation". In: Cerebral Cortex 25.6, pp. 1565-1575.

Candès, Emmanuel and Justin Romberg (2005). $\ell_{1}$ MAGIC: Recovery of sparse signals via convex programming. Tech. rep. http://www. acm. caltech. 
edu/11magic/downloads/11magic.pdf. Stanford, CA: Stanford University.

Cantio, Cathriona et al. (2016). "Exploring 'The autisms' at a cognitive level". In: Autism Research 9.12, pp. 1328-1339.

Casanova, Manuel F (2004). "White matter volume increase and minicolumns in autism". In: Annals of Neurology 56.3, pp. 453-453.

- (2007). "The neuropathology of autism". In: Brain Pathology 17.4, pp. 422-433.

Casanova, Manuel F et al. (2002). "Minicolumnar pathology in autism". In: Neurology 58.3, pp. 428432.

Casanova, Manuel F et al. (2006). "Minicolumnar abnormalities in autism". In: Acta Neuropathologica 112.3, pp. 287-303.

Catani, Marco et al. (2016). "Frontal networks in adults with autism spectrum disorder". In: Brain 139.2, pp. 616-630.

Chen, Nutan et al. (2015). "Efficient movement representation by embedding dynamic movement primitives in deep autoencoders". In: Humanoid Robots (Humanoids), 2015 IEEE-RAS 15th International Conference on. IEEE, pp. 434-440.

Chisholm, Katharine et al. (2015). "The association between autism and schizophrenia spectrum disorders: A review of eight alternate models of co-occurrence". In: Neuroscience \& Biobehavioral Reviews 55, pp. 173183.

Chrobak, James J, András Lőrincz, and György Buzsáki (2000). "Physiological patterns in the hippocampoentorhinal cortex system". In: Hippocampus 10.4, pp. 457-465.

Church, Barbara A et al. (2015). "Learning, plasticity, and atypical generalization in children with autism". In: Psychonomic Bulletin \& Review 22.5, pp. 13421348.

Cocchi, Luca et al. (2017). "Criticality in the brain: A synthesis of neurobiology, models and cognition". In: Progress in Neurobiology 158, pp. 132-152.

Cook, Richard et al. (2014). "Intact facial adaptation in autistic adults". In: Autism Research 7.4, pp. 481490.

Courchesne, Eric and Karen Pierce (2005). "Brain overgrowth in autism during a critical time in development: Implications for frontal pyramidal neuron and interneuron development and connectivity". In: International Journal of Developmental Neuroscience 23.2-3, pp. 153-170.

David, Maude M et al. (2016a). "Comorbid Analysis of Genes Associated with Autism Spectrum Disorders Reveals Differential Evolutionary Constraints". In: PloS One 11.7, e0157937.

David, Nicole, Albert Newen, and Kai Vogeley (2008). "The "sense of agency" and its underlying cognitive and neural mechanisms". In: Consciousness and Cognition 17.2 , pp. 523-534.

David, Nicole et al. (2016b). "Variability of cortical oscillation patterns: A possible endophenotype in autism spectrum disorders?" In: Neuroscience \& Biobehavioral Reviews 71, pp. 590-600.

Davis, Greg and Kate Plaisted-Grant (2015). "Low endogenous neural noise in autism". In: Autism 19.3, pp. 351-362.

De Gardelle, Vincent et al. (2012). "Concurrent repetition enhancement and suppression responses in extrastriate visual cortex". In: Cerebral Cortex 23.9, pp. 2235-2244.

Dehorter, Nathalie et al. (2017). "Tuning neural circuits by turning the interneuron knob". In: Current Opinion in Neurobiology 42, pp. 144-151.

Dekeyzer, Sven et al. (2017). "“Unforgettable" - a pictorial essay on anatomy and pathology of the hippocampus". In: Insights into Imaging 8.2, pp. 199212.

Dennett, Daniel C (1991). Consciousness Explained. Boston (Little, Brown and Co).

Deoni, Sean CL et al. (2016). "White matter maturation profiles through early childhood predict general cognitive ability". In: Brain Structure and Function 221.2, pp. 1189-1203.

Deschrijver, Eliane, Jan R Wiersema, and Marcel Brass (2017). "The influence of action observation on action execution: Dissociating the contribution of action on perception, perception on action, and resolving conflict". In: Cognitive, Affective, \& Behavioral Neuroscience 17.2, pp. 381-393.

Dickinson, Abigail, Myles Jones, and Elizabeth Milne (2016). "Measuring neural excitation and inhibition in autism: Different approaches, different findings and different interpretations". In: Brain Research 1648, pp. 277-289.

Diekelmann, Susanne and Jan Born (2010). "The memory function of sleep". In: Nature Reviews Neuroscience 11.2 , p. 114.

Donoho, David L (2006). "Compressed sensing". In: IEEE Transactions on Information Theory 52.4, pp. 1289-1306.

Dragoi, George and György Buzsáki (2006). “Temporal encoding of place sequences by hippocampal cell assemblies". In: Neuron 50.1, pp. 145-157.

Duan, Dingna et al. (2017). "Longitudinal multi-scale mapping of infant cortical folding using spherical wavelets". In: Biomedical Imaging (ISBI 2017), 2017 IEEE 14th International Symposium on. IEEE, pp. 9396.

Duda, M et al. (2017). "Crowdsourced validation of a machine-learning classification system for autism and ADHD". In: Translational Psychiatry 7.5, e1133.

Ecker, C et al. (2016). "Relationship between cortical gyrification, white matter connectivity, and autism spectrum disorder". In: Cerebral Cortex 26.7, pp. 3297-3309.

Ewbank, Michael P et al. (2014). "Repetition suppression in ventral visual cortex is diminished as a function of increasing autistic traits". In: Cerebral Cortex 25.10, pp. 3381-3393. 
Fernández-Ruiz, Antonio et al. (2017). "EntorhinalCA3 dual-input control of spike timing in the hippocampus by theta-gamma coupling". In: Neuron 93.5, pp. 1213-1226.

Ferrarelli, Fabio et al. (2010). "Thalamic dysfunction in schizophrenia suggested by whole-night deficits in slow and fast spindles". In: American Journal of Psychiatry 167.11, pp. 1339-1348.

Finnegan, Rory, Mark Shaw, and Suzanna Becker (2017). "Restricted Boltzmann Machine Models of Hippocampal Coding and Neurogenesis". In: The Rewiring Brain. Elsevier, pp. 443-461.

Forbes, Thomas A and Vittorio Gallo (2017). "All wrapped up: Environmental effects on myelination". In: Trends in Neurosciences 40.9, pp. 572-587.

Franzius, Mathias, Henning Sprekeler, and Laurenz Wiskott (2007). "Slowness and sparseness lead to place, head-direction, and spatial-view cells". In: PLoS Computational Biology 3.8, e166.

Freund, Tamas F and György Buzsáki (1996). "Interneurons of the hippocampus". In: Hippocampus 6.4, pp. 347-470.

Frith, Uta (1991). "Asperger and his syndrome". In: Autism and Asperger syndrome 14, pp. 1-36.

Frith, Uta and Francesca Happé (1994). "Autism: Beyond "theory of mind"'. In: Cognition 50.1-3, pp. 115-132.

Froehlich, AL et al. (2012). "Intact prototype formation but impaired generalization in autism". In: Research in Autism Spectrum Disorders 6.2, pp. 921-930.

Gallese, Vittorio et al. (1996). "Action recognition in the premotor cortex". In: Brain 119.2, pp. 593-609.

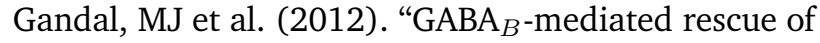
altered excitatory-inhibitory balance, gamma synchrony and behavioral deficits following constitutive NMDAR-hypofunction". In: Translational Psychiatry 2.7, e142.

Gao, R and P Penzes (2015). "Common mechanisms of excitatory and inhibitory imbalance in schizophrenia and autism spectrum disorders". In: Current Molecular Medicine 15.2, pp. 146-167.

Gelinas, Jennifer N et al. (2016). "Interictal epileptiform discharges induce hippocampal-cortical coupling in temporal lobe epilepsy". In: Nature Medicine 22.6, pp. 641-648.

Girardeau, Gabrielle, Ingrid Inema, and György Buzsáki (2017). "Reactivations of emotional memory in the hippocampus-amygdala system during sleep". In: Nature Neuroscience 20.11, pp. 1634-1642.

Graziano, Michael (2006). "The organization of behavioral repertoire in motor cortex". In: Annual Review of Neuroscience 29, pp. 105-134.

Grützner, Christine et al. (2013). "Deficits in high-(> $60 \mathrm{~Hz}$ ) gamma-band oscillations during visual processing in schizophrenia". In: Frontiers in Human Neuroscience 7, p. 88.

Happé, Francesca and Uta Frith (2006). "The weak coherence account: Detail-focused cognitive style in autism spectrum disorders". In: Journal of Autism and Developmental Disorders 36.1, pp. 5-25.

Harris, Julia J, Renaud Jolivet, and David Attwell (2012). "Synaptic energy use and supply". In: Neuron 75.5, pp. 762-777.

Harris, Zellig S (1954). "Distributional structure". In: Word Journal of The International Linguistic Association 10.2-3, pp. 146-162.

Hashemi, Ezzat et al. (2017). "The number of parvalbumin-expressing interneurons is decreased in the medial prefrontal cortex in autism". In: Cerebral Cortex 27.3, pp. 1931-1943.

Hazlett, Heather Cody et al. (2017). "Early brain development in infants at high risk for autism spectrum disorder". In: Nature 542.7641, pp. 348-351.

Hedenius, Martina et al. (2011). "Grammar predicts procedural learning and consolidation deficits in children with specific language impairment". In: Research in Developmental Disabilities 32.6, pp. 23622375.

Heindel, William C et al. (2013). "Prototype learning and dissociable categorization systems in Alzheimer's disease". In: Neuropsychologia 51.9, pp. 1699-1708.

Henze, Darrell A, Lucia Wittner, and György Buzsáki (2002). "Single granule cells reliably discharge targets in the hippocampal CA3 network in vivo". In: Nature Neuroscience 5.8, pp. 790-795.

Herry, Cyril et al. (2008). "Switching on and off fear by distinct neuronal circuits". In: Nature 454.7204, pp. 600-606.

Hills, Peter James (2013). "Aftereffects in face processing". In: Frontiers in Psychology 4, p. 854.

Hinton, Geoffrey E et al. (1995). "The "wake-sleep" algorithm for unsupervised neural networks". In: Science 268.5214, pp. 1158-1161.

Holmes, Gregory L (2016). "Effect of seizures on the developing brain and cognition". In: Seminars in Pediatric Neurology. Vol. 23. 2. Elsevier, pp. 120126.

Hong, L Elliot et al. (2010). "Gamma and delta neural oscillations and association with clinical symptoms under subanesthetic ketamine". In: Neuropsychopharmacology 35.3, pp. 632-640.

Horton, Jonathan C and Daniel L Adams (2005). "The cortical column: A structure without a function". In: Philosophical Transactions of the Royal Society of London B: Biological Sciences 360.1456, pp. 837-862.

Howarth, Clare, Padraig Gleeson, and David Attwell (2012). "Updated energy budgets for neural computation in the neocortex and cerebellum". In: Journal of Cerebral Blood Flow \& Metabolism 32.7, pp. 12221232.

Hughes, James EA et al. (2017). "Is synaesthesia more prevalent in autism spectrum conditions? Only where there is prodigious talent". In: Multisensory Research 30.3-5, pp. 391-408. 
Hughes, John R (2007). "Autism: The first firm finding = underconnectivity?" In: Epilepsy \& Behavior 11.1 , pp. 20-24.

Ijspeert, Auke Jan et al. (2013). "Dynamical movement primitives: Learning attractor models for motor behaviors". In: Neural Computation 25.2, pp. 328-373.

Inan, Melis et al. (2016). "Energy deficit in parvalbumin neurons leads to circuit dysfunction, impaired sensory gating and social disability". In: Neurobiology of Disease 93, pp. 35-46.

Innocenti, Giorgio M, Alessandro Vercelli, and Roberto Caminiti (2013). "The diameter of cortical axons depends both on the area of origin and target". In: Cerebral Cortex 24.8, pp. 2178-2188.

Javitt, Daniel C (2009). "When doors of perception close: Bottom-up models of disrupted cognition in schizophrenia". In: Annual Review of Clinical Psychology 5, pp. 249-275.

Kaiser, Marcus (2017). "Mechanisms of connectome development". In: Trends in Cognitive Sciences 21.9, pp. 703-717.

Kann, Oliver (2012). "The energy demand of fast neuronal network oscillations: Insights from brain slice preparations". In: Frontiers in Pharmacology 2, p. 90.

Kanner, Leo (1943). "Autistic disturbances of affective contact". In: Nervous Child 2.3, pp. 217-250.

Kates, Wendy R, Ichiro Ikuta, and Courtney P Burnette (2009). "Gyrification patterns in monozygotic twin pairs varying in discordance for autism". In: Autism Research 2.5, pp. 267-278.

Kaur, Maninderjit, Sudha M Srinivasan, and Anjana $\mathrm{N}$ Bhat (2018). "Comparing motor performance, praxis, coordination, and interpersonal synchrony between children with and without Autism Spectrum Disorder (ASD)". In: Research in Developmental Disabilities 72, pp. 79-95.

Kearns, Michael and Daphne Koller (1999). "Efficient reinforcement learning in factored MDPs". In: International Joint Conference on Artificial Intelligence. Vol. 16, pp. 740-747.

Kéri, Szabolcs et al. (2001). "Are Alzheimer's disease patients able to learn visual prototypes?" In: Neuropsychologia 39.11, pp. 1218-1223.

Kéri, Szabolcs et al. (2002). "Categories, prototypes and memory systems in Alzheimer's disease". In: Trends in Cognitive Sciences 6.3, pp. 132-136.

Khodagholy, Dion, Jennifer N Gelinas, and György Buzsáki (2017). "Learning-enhanced coupling between ripple oscillations in association cortices and hippocampus". In: Science 358.6361, pp. 369-372.

Knowlton, Barbara J and Larry R Squire (1993). "The learning of categories: Parallel brain systems for item memory and category knowledge". In: Science 262.5140, pp. 1747-1749.

Kovács, Gyula et al. (2007). "Adaptation duration affects the spatial selectivity of facial aftereffects". In: Vision Research 47.25, pp. 3141-3149.
Kovács, Ilona (2000). "Human development of perceptual organization". In: Vision Research 40.10-12, pp. 1301-1310.

Kovács, Ilona et al. (1999). "Late maturation of visual spatial integration in humans". In: Proceedings of the National Academy of Sciences 96.21, pp. 12204 12209.

Krishnan, Arjun et al. (2016). "Genome-wide prediction and functional characterization of the genetic basis of autism spectrum disorder". In: Nature Neuroscience 19.11, pp. 1454-1462.

Kuijper, Sanne JM et al. (2017). "Narrative production in children with autism spectrum disorder (ASD) and children with attention-deficit/hyperactivity disorder (ADHD): Similarities and differences." In: Journal of Abnormal Psychology 126.1, pp. 63-75.

Larson, Felicity V et al. (2017). "Psychosis in autism: Comparison of the features of both conditions in a dually affected cohort". In: The British Journal of Psychiatry 210.4, pp. 269-275.

Lawson, Rebecca P, Geraint Rees, and Karl J Friston (2014). "An aberrant precision account of autism". In: Frontiers in Human Neuroscience 8, p. 302.

Lee, Eun-Jae, Su Yeon Choi, and Eunjoon Kim (2015). "NMDA receptor dysfunction in autism spectrum disorders". In: Current Opinion in Pharmacology 20, pp. 8-13.

Lee, Tai Sing and David Mumford (2003). "Hierarchical Bayesian inference in the visual cortex". In: Journal of the Optical Society of America A 20.7, pp. 14341448.

Lemaitre, Anne-Laure, Marion Luyat, and Gilles Lafargue (2016). "Individuals with pronounced schizotypal traits are particularly successful in tickling themselves". In: Consciousness and Cognition 41, pp. 6471.

Lemonnier, E et al. (2017). "Effects of bumetanide on neurobehavioral function in children and adolescents with autism spectrum disorders". In: Translational Psychiatry 7.3, e1056.

Leopold, David A and Nikos K Logothetis (1996). "Activity changes in early visual cortex reflect monkeys' percepts during binocular rivalry". In: Nature 379.6565, pp. 549-553.

Levina, Anna and Viola Priesemann (2017). "Subsampling scaling". In: Nature Communications 8, p. 15140 .

Lewicki, Michael S (2002). "Efficient coding of natural sounds". In: Nature Neuroscience 5.4, pp. 356-363.

Lewis, David A, Takanori Hashimoto, and David W Volk (2005). "Cortical inhibitory neurons and schizophrenia". In: Nature Reviews Neuroscience 6.4, pp. 312324.

Lewis, John D et al. (2017). "The emergence of network inefficiencies in infants with autism spectrum disorder". In: Biological Psychiatry 82.3, pp. 176185.

Lisman, John and György Buzsáki (2008). "A neural coding scheme formed by the combined function 
of gamma and theta oscillations". In: Schizophrenia Bulletin 34.5, pp. 974-980.

Lisman, John E and Ole Jensen (2013). "The thetagamma neural code". In: Neuron 77.6, pp. 10021016.

Lo-Castro, Adriana and Paolo Curatolo (2014). "Epilepsy associated with autism and attention deficit hyperactivity disorder: Is there a genetic link?" In: Brain and Development 36.3, pp. 185-193.

Locke, John L (1995). The child's path to spoken language. Harvard University Press.

Lőrincz, A et al. (2018). "Towards reasoning based representations: Deep Consistence Seeking Machine”. In: Cognitive Systems Research 47, pp. 92-108.

Lőrincz, András and György Buzsáki (2000). "Twophase computational model training long-term memories in the entorhinal-hippocampal region". In: Annals of the New York Academy of Sciences 911.1, pp. 83-111.

Lőrincz, András and András Sárkány (2017). “SemiSupervised Learning of Cartesian Factors: A TopDown Model of the Entorhinal Hippocampal Complex". In: Frontiers in Psychology 8, p. 215.

Lőrincz, András, Botond Szatmáry, and Gábor Szirtes (2002). "The mystery of structure and function of sensory processing areas of the neocortex: A resolution". In: Journal of Computational Neuroscience 13.3, pp. 187-205.

Lőrincz, András and Gábor Szirtes (2009). "Here and now: How time segments may become events in the hippocampus". In: Neural Networks 22.5-6, pp. 738747.

Lőrincz, András et al. (2002). “Ockham's Razor at Work: Modeling of the"Homunculus"'. In: Brain and Mind 3.2, pp. 187-220.

Lőrincz, András et al. (2016). "Columnar machine: Fast estimation of structured sparse codes". In: Biologically Inspired Cognitive Architectures 15, pp. 19-33.

Loth, Eva, Declan G Murphy, and Will Spooren (2016). "Defining precision medicine approaches to autism spectrum disorders: concepts and challenges". In: Frontiers in Psychiatry 7, p. 188.

Mandy, William and Meng-Chuan Lai (2016). "Annual research review: The role of the environment in the developmental psychopathology of autism spectrum condition". In: Journal of Child Psychology and Psychiatry 57.3, pp. 271-292.

Marchese, Maria et al. (2014). "Autism-epilepsy phenotype with macrocephaly suggests PTEN, but not GLIALCAM, genetic screening". In: BMC Medical Genetics 15.1, p. 26.

Markram, Henry, Tania Rinaldi, and Kamila Markram (2007). "The intense world syndrome-an alternative hypothesis for autism". In: Frontiers in Neuroscience 1, p. 6.

Mazarati, Andrey M, Megan L Lewis, and Quentin J Pittman (2017). "Neurobehavioral comorbidities of epilepsy: Role of inflammation". In: Epilepsia 58.S3, pp. 48-56.
McAuliffe, Danielle et al. (2017). "Dyspraxia in ASD: Impaired coordination of movement elements". In: Autism Research 10.4, pp. 648-652.

McGurk, Harry and John MacDonald (1976). "Hearing lips and seeing voices". In: Nature 264.5588, pp. 746-748.

McKavanagh, Rebecca, Eleanor Buckley, and Steven A Chance (2015). "Wider minicolumns in autism: A neural basis for altered processing?" In: Brain 138.7, pp. 2034-2045.

Melloni, Lucia et al. (2007). "Synchronization of neural activity across cortical areas correlates with conscious perception". In: Journal of Neuroscience 27.11, pp. 2858-2865.

Morgan, Michael John, K Schreiber, and Joshua Adam Solomon (2016). "Low-level mediation of directionally specific motion aftereffects: Motion perception is not necessary". In: Attention, Perception, \& Psychophysics 78.8, pp. 2621-2632.

Moser, Edvard I, May-Britt Moser, and Bruce L McNaughton (2017). "Spatial representation in the hippocampal formation: A history". In: Nature Neuroscience 20.11, pp. 1448-1464.

Mountcastle, Vernon B (1997). "The columnar organization of the neocortex." In: Brain: a Journal of Neurology 120.4, pp. 701-722.

Németh, Dezső et al. (2010). "Learning in autism: Implicitly superb”. In: PloS One 5.7, e11731.

Noel, Jean-Paul et al. (2017). "The spatial self in schizophrenia and autism spectrum disorder". In: Schizophrenia Research 179, pp. 8-12.

Noel, Jean-Paul et al. (2018). "Disrupted integration of exteroceptive and interoceptive signaling in autism spectrum disorder". In: Autism Research 11.1, pp. 194-205.

Nordahl, Christine Wu et al. (2012). "Increased rate of amygdala growth in children aged 2 to 4 years with autism spectrum disorders: A longitudinal study". In: Archives of General Psychiatry 69.1, pp. 53-61.

Oberman, Lindsay M et al. (2005). "EEG evidence for mirror neuron dysfunction in autism spectrum disorders". In: Cognitive Brain Research 24.2, pp. 190 198.

Ognjanovski, Nicolette et al. (2017). "Parvalbuminexpressing interneurons coordinate hippocampal network dynamics required for memory consolidation". In: Nature Communications 8, p. 15039.

O'Keefe, John and Neil Burgess (1996). "Geometric determinants of the place fields of hippocampal neurons". In: Nature 381.6581, pp. 425-428.

Okruszek, Łukasz et al. (2017). "Social cognition in neuropsychiatric populations: A comparison of theory of mind in schizophrenia and mesial temporal lobe epilepsy". In: Scientific Reports 7.1, p. 484.

Olshausen, Bruno A and David J Field (1996). "Emergence of simple-cell receptive field properties by learning a sparse code for natural images". In: Nature 381.6583, pp. 607-609. 
O'Keefe, John and Lynn Nadel (1978). The hippocampus as a cognitive map. Oxford: Clarendon Press.

Page, Stephanie Cerceo et al. (2018). "The schizophrenia and autism associated gene, transcription factor 4 regulates the columnar distribution of layer $2 / 3$ prefrontal pyramidal neurons in an activitydependent manner". In: Molecular Psychiatry 23.2, p. 304.

Palmer, Colin J, Rebecca P Lawson, and Jakob Hohwy (2017). "Bayesian approaches to autism: Towards volatility, action, and behavior". In: Psychological Bulletin 143.5, p. 521.

Pellicano, Elizabeth and David Burr (2012). "When the world becomes 'too real': A Bayesian explanation of autistic perception”. In: Trends in Cognitive Sciences 16.10, pp. 504-510.

Penzes, Peter et al. (2011). "Dendritic spine pathology in neuropsychiatric disorders". In: Nature Neuroscience 14.3, pp. 285-293.

Peterson, Erik J et al. (2017). " $1 /$ f neural noise is a better predictor of schizophrenia than neural oscillations". In: bioRxiv, p. 113449.

Pina-Camacho, Laura, Mara Parellada, and Marinos Kyriakopoulos (2016). "Autism spectrum disorder and schizophrenia: Boundaries and uncertainties". In: BJ Psych Advances 22.5, pp. 316-324.

Pintér, Balázs et al. (2015). "Wikifying novel words to mixtures of Wikipedia senses by structured sparse coding". In: Pattern Recognition Applications and Methods. Springer, pp. 241-255.

Pitkänen, Asla et al. (2000). "Reciprocal connections between the amygdala and the hippocampal formation, perirhinal cortex, and postrhinal cortex in rat: A review". In: Annals of the New York Academy of Sciences 911.1, pp. 369-391.

Piven, Joseph, Jed T Elison, and Mark J Zylka (2017). "Toward a conceptual framework for early brain and behavior development in autism". In: Molecular Psychiatry 22.10, pp. 1385-1394.

Plaisted, Kate C (2000). "Aspects of autism that theory of mind cannot explain". In: Understanding other minds: Perspectives from developmental cognitive neuroscience, pp. 222-250.

- (2001). "Reduced generalization in autism: An alternative to weak central coherence". In: The development of Autism: Perspectives from Theory and Research. Lawrence Erlbaum Associates, pp. 149-169.

Polepalli, Jai S et al. (2017). "Modulation of excitation on parvalbumin interneurons by neuroligin-3 regulates the hippocampal network". In: Nature Neuroscience 20.2, pp. 219-229.

Posner, Michael I, Ralph Goldsmith, and Kenneth E Welton Jr (1967). "Perceived distance and the classification of distorted patterns". In: Journal of Experimental Psychology 73.1, pp. 28-38.

Pulvermüller, Friedemann (2018). "Neural reuse of action perception circuits for language, concepts and communication". In: Progress in Neurobiology 160, pp. 1-44.
Ramachandran, Vilayanur S and Lindsay M Oberman (2006). "Broken mirrors: A theory of autism". In: Scientific American 295.5, pp. 62-69.

Ramachandran, VS and Elizabeth Seckel (2015). "Synesthetic colors induced by graphemes that have not been consciously perceived". In: Neurocase 21.2, pp. 216-219.

Ramtekkar, Ujjwal P (2017). "DSM-5 Changes in Attention Deficit Hyperactivity Disorder and Autism Spectrum Disorder: Implications for Comorbid Sleep Issues". In: Children 4.8, p. 62.

Rasch, Björn et al. (2007). "Odor cues during slowwave sleep prompt declarative memory consolidation". In: Science 315.5817, pp. 1426-1429.

Renner, Peggy, Laura Grofer Klinger, and Mark R Klinger (2000). "Implicit and explicit memory in autism: Is autism an amnesic disorder?" In: Journal of Autism and Developmental Disorders 30.1, pp. 314.

Ring, Melanie, Sebastian B Gaigg, and Dermot M Bowler (2015). "Object-location memory in adults with autism spectrum disorder". In: Autism Research 8.5, pp. 609-619.

Ring, Melanie et al. (2017). "Structural learning difficulties implicate altered hippocampal functioning in adults with autism spectrum disorder." In: Journal of Abnormal Psychology 126.6, pp. 793-804.

Rizzolatti, Giacomo et al. (1996). "Premotor cortex and the recognition of motor actions". In: Cognitive Brain Research 3.2, pp. 131-141.

Robertson, Caroline E and Simon Baron-Cohen (2017). "Sensory perception in autism". In: Nature Reviews Neuroscience 18.11, pp. 671-684.

Robertson, Caroline E et al. (2013). "Slower rate of binocular rivalry in autism". In: Journal of Neuroscience 33.43, pp. 16983-16991.

Rothwell, Patrick E et al. (2014). "Autism-associated neuroligin-3 mutations commonly impair striatal circuits to boost repetitive behaviors". In: Cell 158.1, pp. 198-212.

Rouw, Romke and H Steven Scholte (2007). "Increased structural connectivity in grapheme-color synesthesia". In: Nature Neuroscience 10.6, pp. 792-797.

Roux, Lisa et al. (2017). "Sharp wave ripples during learning stabilize the hippocampal spatial map". In: Nature neuroscience 20.6, pp. 845-853.

Rowland, David C et al. (2016). "Ten years of grid cells". In: Annual Review of Neuroscience 39, pp. 1940.

Rubenstein, JLR and Michael M Merzenich (2003). "Model of autism: Increased ratio of excitation/inhibition in key neural systems". In: Genes, Brain and Behavior 2.5, pp. 255-267.

Rubinstein, Ron, Michael Zibulevsky, and Michael Elad (2010). "Double sparsity: Learning sparse dictionaries for sparse signal approximation". In: IEEE Transactions on Signal Processing 58.3, pp. 1553-1564. 
Ruderman, Daniel L (1994). "The statistics of natural images". In: Network: Computation in Neural Systems 5.4 , pp. $517-548$.

Rutter, Michael (1972). "Childhood schizophrenia reconsidered". In: Journal of Autism and Childhood Schizophrenia 2.3, pp. 315-337.

Schaal, Stefan (2006). "Dynamic movement primitivesa framework for motor control in humans and humanoid robotics". In: Adaptive Motion of Animals and Machines. Springer, pp. 261-280.

Schmitz, Taylor W et al. (2017). "Hippocampal GABA enables inhibitory control over unwanted thoughts". In: Nature Communications 8.1, p. 1311.

Schuetze, Manuela et al. (2017). "Reinforcement Learning in Autism Spectrum Disorder". In: Frontiers in Psychology 8.

Schwartz, Odelia and Eero P Simoncelli (2001). "Natural signal statistics and sensory gain control". In: Nature Neuroscience 4.8, pp. 819-825.

Searle, John R (1992). The rediscovery of the mind. MIT press.

- (2013). "Is the brain a digital computer?" In: The American Philosophical Association Centennial Series, pp. 691-710.

Selfe, Lorna (1977). Nadia: A case of extraordinary drawing ability in an autistic child. Academic Press.

- (2012). Nadia revisited: A longitudinal study of an Autistic Savant. Psychology Press.

Siegel, Jerome M (2005). "Clues to the functions of mammalian sleep". In: Nature 437.7063, pp. 1264 1271.

Singer, Wolf (2013). "Cortical dynamics revisited". In: Trends in Cognitive Sciences 17.12, pp. 616-26.

Snyder, Allan W and Mandy Thomas (2001). "The paradox of autistic art". In: Artlink 21.2, pp. 48-50.

Sowden, Sophie et al. (2016). "Intact automatic imitation and typical spatial compatibility in autism spectrum disorder: Challenging the broken mirror theory". In: Autism Research 9.2, pp. 292-300.

Spengler, Stephanie, Geoffrey Bird, and Marcel Brass (2010). "Hyperimitation of actions is related to reduced understanding of others' minds in autism spectrum conditions". In: Biological Psychiatry 68.12, pp. 1148-1155.

Spocter, Muhammad A et al. (2015). "The Minicolumn in Comparative Context". In: Recent Advances on the Modular Organization of the Cortex. Springer, pp. 6380.

Sprechmann, Pablo, Alexander M Bronstein, and Guillermo Sapiro (2015). "Learning efficient sparse and low rank models". In: IEEE Transactions on Pattern Analysis and Machine Intelligence 37.9, pp. 18211833.

Squire, Larry R and Barbara J Knowlton (1995). "Learning about categories in the absence of memory". In: Proceedings of the National Academy of Sciences 92.26, pp. 12470-12474.

Srivastava, Siddharth and Mustafa Sahin (2017). "Autism spectrum disorder and epileptic en- cephalopathy: Common causes, many questions". In: Journal of Neurodevelopmental Disorders 9.1, p. 23.

Stickgold, Robert and Matthew P Walker (2005). "Sleep and memory: The ongoing debate". In: Sleep 28.10, pp. 1225-1227.

Subramanian, Krishna et al. (2017). "Basal ganglia and autism - a translational perspective". In: Autism Research 10, pp. 1751-1775.

Szatmari, Peter et al. (2007). "Mapping autism risk loci using genetic linkage and chromosomal rearrangements". In: Nature Genetics 39.3, pp. 319-328.

Szita, István and András Lőrincz (2009). “Optimistic initialization and greediness lead to polynomial time learning in factored MDPs". In: Proceedings of the 26th Annual International Conference on Machine Learning. ACM, pp. 1001-1008.

Taube, Jeffrey S (2007). "The head direction signal: Origins and sensory-motor integration". In: Annual Review of Neuroscience 30, pp. 181-207.

Taube, Jeffrey S, Robert U Muller, and James B Ranck (1990). "Head-direction cells recorded from the postsubiculum in freely moving rats. I. Description and quantitative analysis". In: Journal of Neuroscience 10.2, pp. 420-435.

Tek, Saime et al. (2014). "Longitudinal analyses of expressive language development reveal two distinct language profiles among young children with autism spectrum disorders". In: Journal of Autism and Developmental Disorders 44.1, pp. 75-89.

Toro, Roberto et al. (2010). "Key role for gene dosage and synaptic homeostasis in autism spectrum disorders". In: Trends in Genetics 26.8, pp. 363-372.

Uhlhaas, Peter J and Wolf Singer (2006). "Neural synchrony in brain disorders: Relevance for cognitive dysfunctions and pathophysiology". In: Neuron 52.1, pp. 155-168.

- (2012). "Neuronal dynamics and neuropsychiatric disorders: Toward a translational paradigm for dysfunctional large-scale networks". In: Neuron 75.6, pp. 963-980.

Uhlhaas, Peter J et al. (2006). "Dysfunctional longrange coordination of neural activity during Gestalt perception in schizophrenia". In: Journal of Neuroscience 26.31, pp. 8168-8175.

Ujiie, Yuta et al. (2015). "The McGurk effect and autistic traits; An analogue perspective". In: Letters on Evolutionary Behavioral Science 6.2, pp. 9-12.

Valvo, Giulia et al. (2016). "Temporal lobe connects regression and macrocephaly to autism spectrum disorders". In: European Child \& Adolescent Psychiatry 25.4, pp. 421-429.

Van Belle, Goedele et al. (2010). "Whole not hole: Expert face recognition requires holistic perception". In: Neuropsychologia 48.9, pp. 2620-2629.

Van Doorn, George, Jakob Hohwy, and Mark Symmons (2014). "Can you tickle yourself if you swap bodies with someone else?" In: Consciousness and Cognition 23, pp. 1-11. 
Colors of Autism Spectrum:

Vincent, Pascal et al. (2008). "Extracting and composing robust features with denoising autoencoders". In: Proceedings of the 25th International Conference on Machine Learning. ACM, pp. 1096-1103.

Vinogradova, OS (1970). "Registration of information and the limbic system". In: Short-term changes in neural activity and behavior. Cambridge University Press Cambridge, pp. 95-140.

Von Der Lühe, Tabea et al. (2016). "Interpersonal predictive coding, not action perception, is impaired in autism". In: Philosophical Transactions of the Royal Society B 371.1693, p. 2015.0373.

Ward, Jamie et al. (2017). "An autistic-like profile of attention and perception in synaesthesia". In: Cortex. in press.

Weir, RK et al. (2018). "Protracted dendritic growth in the typically developing human amygdala and increased spine density in young ASD brains". In: Journal of Comparative Neurology 526.2, pp. 262274.

Willfors, C et al. (2017). "Medical history of discordant twins and environmental etiologies of autism". In: Translational Psychiatry 7.1, e1014.

Williams, Justin HG et al. (2001). "Imitation, mirror neurons and autism". In: Neuroscience \& Biobehavioral Reviews 25.4, pp. 287-295.

Winterer, Georg (2017). "Noisy networks and autism". In: Biological Psychiatry 82.3, pp. 152-154.

Winterer, Georg et al. (2004). "Prefrontal broadband noise, working memory, and genetic risk for schizophrenia". In: American Journal of Psychiatry 161.3, pp. 490-500.

Witter, Menno P, Heidi Kleven, and Asgeir Kobro Flatmoen (2017). "Comparative contemplations on the hippocampus". In: Brain, Behavior and Evolution 90.1, pp. 15-24.

Witter, Menno P et al. (2014). "Architecture of spatial circuits in the hippocampal region". In: Philosophical Transactions of the Royal Society B 369.1635, p. 20120515.

Woynaroski, Tiffany G et al. (2013). "Multisensory speech perception in children with autism spectrum disorders". In: Journal of Autism and Developmental Disorders 43.12, pp. 2891-2902.

$\mathrm{Xu}$, Chun et al. (2016). "Distinct hippocampal pathways mediate dissociable roles of context in memory retrieval". In: Cell 167.4, pp. 961-972.

Xue, Gui et al. (2011). "Spaced learning enhances subsequent recognition memory by reducing neural repetition suppression". In: Journal of Cognitive Neuroscience 23.7, pp. 1624-1633.

Yáñez, Inmaculada Ballesteros et al. (2005). "Double bouquet cell in the human cerebral cortex and a comparison with other mammals". In: Journal of Comparative Neurology 486.4, pp. 344-360.

Yartsev, Michael M and Nachum Ulanovsky (2013). "Representation of three-dimensional space in the hippocampus of flying bats". In: Science 340.6130, pp. 367-372.
Yoshida, Ririko et al. (2017). "Dynamics of gyrification in the human cerebral cortex during development". In: Congenital Anomalies 57.1, pp. 8-14.

Zwart, Fenny S et al. (2017). "Procedural learning across the lifespan: A systematic review with implications for atypical development". In: Journal of Neuropsychology. 